\title{
THE STRUCTURE OF HYPERFINITE BOREL EQUIVALENCE RELATIONS
}

\author{
R. DOUGHERTY, S. JACKSON, AND A. S. KECHRIS
}

\begin{abstract}
We study the structure of the equivalence relations induced by the orbits of a single Borel automorphism on a standard Borel space. We show that any two such equivalence relations which are not smooth, i.e., do not admit Borel selectors, are Borel embeddable into each other. (This utilizes among other things work of Effros and Weiss.) Using this and also results of Dye, Varadarajan, and recent work of Nadkarni, we show that the cardinality of the set of ergodic invariant measures is a complete invariant for Borel isomorphism of aperiodic nonsmooth such equivalence relations. In particular, since the only possible such cardinalities are the finite ones, countable infinity, and the cardinality of the continuum, there are exactly countably infinitely many isomorphism types. Canonical examples of each type are also discussed.
\end{abstract}

This paper is a contribution to the study of Borel equivalence relations on standard Borel spaces. We concentrate here on the study of the hyperfinite ones. These are by definition the increasing unions of sequences of Borel equivalence relations with finite equivalence classes but equivalently they can be also described as the ones induced by the orbits of a single Borel automorphism. They include therefore a great variety of examples, some of them discussed in $\S 6$. For instance, the equivalence relations: $E_{0}$ on $2^{\mathbb{N}}$ (where $x E_{0} y$ iff $x, y$ are eventually equal, i.e., $\left.\exists n \forall m \geq n\left(x_{m}=y_{m}\right)\right), E_{t}$ on $2^{\mathbb{N}}$ (where $x E_{t} y$ iff $x, y$ have equal tails, i.e., $\left.\exists n \exists m \forall k\left(x_{n+k}=y_{m+k}\right)\right), E(\mathbb{Z}, 2)$ on $2^{\mathbb{Z}}$ (where $x E(\mathbb{Z}, 2) y$ iff $x$ is a shift of $y$ ), $E_{\alpha}$ on the unit circle $\mathbb{T}$ (where $\alpha \in \mathbb{T}$ and $x E_{\alpha} y$ iff $x$ is the rotation of $y$ by $n \alpha, n \in \mathbb{Z}), E(\mathbb{R} / \mathbb{Q}$ ) on $\mathbb{R}$ (the Vitali equivalence relation, i.e., $x E(\mathbb{R} / \mathbb{Q}) y$ iff $x-y \in \mathbb{Q})$, are all hyperfinite.

Our main results in this paper provide a classification of hyperfinite Borel equivalence relations under two different notions of equivalence. The weaker one, which we call bi-embeddability, is the following: Given hyperfinite Borel equivalence relations $E, F$ (on $X, Y$ resp.) we say that $E$ embeds into $F$, in symbols $E \sqsubseteq F$, if there is a Borel injection $f: X \rightarrow Y$ such that $x E y \Leftrightarrow$ $f(x) F f(y)$. Then $E, F$ are bi-embeddable, in symbols $E \approx F$, if $E \sqsubseteq F$ and $F \sqsubseteq E$. As it turns out, except for the trivial class of smooth relations, i.e., those having Borel selectors, any two hyperfinite Borel equivalence relations are bi-embeddable; i.e., we have

Received by the editors September 24, 1991.

1991 Mathematics Subject Classification. Primary 28D05; Secondary 03E15.

Research of the authors was partially supported by NSF grants DMS-9158092 (R.D.), DMS9007808 (S.J.) and DMS-9020153 (A.S.K.). 
Theorem 1. Let $E, F$ be nonsmooth hyperfinite Borel equivalence relations. Then $E \approx F$.

The strong notion of equivalence that we consider next is that of Borel isomorphism, where $E, F$ (on $X, Y$ resp.) are Borel isomorphic, in symbols $E \cong F$, if there is a Borel bijection $f: X \rightarrow Y$ with $x E y \Leftrightarrow f(x) F f(y)$. By using Theorem 1 and recent work of Nadkarni [N2], as well as some classical results in ergodic theory, such as Dye's Theorem and the Ergodic Decomposition Theorem, we are able to classify completely hyperfinite Borel equivalence relations up to Borel isomorphism. For the nontrivial case of the aperiodic ones, i.e. those containing no finite equivalence classes, this works as follows:

Theorem 2. Let $E$ be an aperiodic, nonsmooth hyperfinite Borel equivalence relation. Then $E$ is Borel isomorphic to exactly one of the following. $E_{t}, E_{0} \times$ $\Delta(n)$ (the product of $E_{0}$ with the equality relation on $n$ elements) for $1 \leq n \leq$ $\aleph_{0}, E^{*}(\mathbb{Z}, 2)$ (the restriction of $E(\mathbb{Z}, 2)$ to the aperiodic points of $2^{\mathbb{Z}}$ ).

This theorem is equivalent to the following result providing a complete invariant for Borel isomorphism. Given a hyperfinite Borel equivalence relation $E$ on $X$, induced by a Borel automorphism $T$, we say that a probability measure $\mu$ on $X$ is $E$-invariant if it is $T$-invariant and $E$-ergodic if it is $T$-ergodic (i.e. every $T$-invariant set has measure 0 or 1$)$. It is easy to check that this definition does not depend on $T$. Denote by $\mathscr{E}_{0}(E)$ the space of nonatomic (this is unnecessary if $E$ is aperiodic), $E$-invariant, ergodic measures. Then we have

Theorem $2^{\prime}$. The cardinal number card $\left(\mathscr{E}_{0}(E)\right)$ is a complete invariant for Borel isomorphism of aperiodic, nonsmooth hyperfinite Borel equivalence relations, i.e. for any two such $E, F$,

$$
E \cong F \Leftrightarrow \operatorname{card}\left(\mathscr{E}_{0}(E)\right)=\operatorname{card}\left(\mathscr{E}_{0}(F)\right) .
$$

This was conjectured by M. G. Nadkarni (see [CN2]), who proved first in [N3] the case when $\operatorname{card}\left(\mathscr{E}_{0}(E)\right)=\operatorname{card}\left(\mathscr{E}_{0}(F)\right)$ is countable, by using his result in [N2] and Theorem 1.

This paper is organized as follows. In $\S 1$ we discuss in general countable (i.e., having countable equivalence classes) Borel equivalence relations, review a representation result of Feldman-Moore [FM], and discuss some of its consequences. In $\S 2$, we study the well-known notion of compressibility that plays an important role in the sequel. In $\S \S 3$ and 4 we present some basic facts about invariant and quasi-invariant measures. The notion of hyperfinite Borel equivalence relation is discussed in $\S 5$, and in $\S 6$ various examples are presented. In $\S 7$, we prove Theorem 1 and some of its consequences. The hyperfiniteness of tail equivalence relations is established in $\S 8$. The classification Theorems 2 and $2^{\prime}$ are proved in $\S 9$, and $\S 10$ deals with an illustrative class of examplesthe Lipschitz automorphisms of $2^{\mathbb{N}}$. Finally, $\S 11$ collects miscellaneous facts related to the results in this paper and other work in the literature.

There are several interesting open problems concerning hyperfiniteness. For example:

(1) Is the increasing union of a sequence of hyperfinite Borel equivalence relations hyperfinite?

(2) (Weiss) Is an equivalence relation induced by a Borel action of a countable amenable (or even abelian) group hyperfinite? (This is known to be true for the groups $\mathbb{Z}^{n}$-Weiss.) 
(3) Is the notion of hyperfiniteness effective, i.e., if $E$ is a $\Delta_{1}^{1}$ hyperfinite equivalence relation on $2^{\mathbb{N}}$, is $E$ induced by a $\Delta_{1}^{1}$ automorphism of $2^{\mathbb{N}}$ ?

There is an extensive literature on the subject of countable Borel equivalence relations in a measure theoretic framework, as it relates to both ergodic theory and the theory of operator algebras. The reader can consult the surveys C. C. Moore [Mo] and K. Schmidt [S2] about this. There is also some recent work in the Baire category framework; see Sullivan-Weiss-Wright [SWW]. In the descriptive set theoretic Borel context that we are interested in, relevant to us here is the work of Weiss [W2, W3], Chaube-Nadkarni [CN1, CN2], Nadkarni [N1, N2, N3], Wagh [Wa] as well as [K1, K2, K3], while further references can be found in the bibliography of these papers. Finally, for standard results in classical descriptive set theory that we use in this paper, see [Ku] or [Mos]. (Two particular such results that are used often below are: The image of a Borel set under a countable-to-1 Borel function is Borel [Mos, 4F.6]; a $G_{\delta}$ subset of a Polish space is Polish [Ku, $\S 33, \mathrm{VI}]$.)

In conclusion, we would like to thank both B. Weiss and M. G. Nadkarni, for helpful discussions and correspondence on the subject matter of this paper.

\section{Countable Borel equivalence Relations}

Let $X$ be a standard Borel space, i.e. a set equipped with a $\sigma$-algebra (its Borel sets) which is Borel isomorphic to the $\sigma$-algebra of the Borel sets in a Polish space. A Borel equivalence relation $E$ on $X$ is an equivalence relation which is Borel as a subset of $X^{2}$ (with the product Borel structure). For each Borel subset $Y \subseteq X$ we denote by $E \uparrow Y:=E \cap Y^{2}$ the restriction of $E$ to $Y$.

We want to consider some basic comparability relations among Borel equivalence relations.

Let $(X, E),\left(X^{\prime}, E^{\prime}\right)$ be two Borel equivalence relations.

(i) We say that $E$ is (Borel) reducible to $E^{\prime}$, in symbols $E \leq E^{\prime}$, iff there is Borel $f: X \rightarrow X^{\prime}$ such that $E=f^{-1}\left[E^{\prime}\right]$, i.e., $x E y \Leftrightarrow f(x) E^{\prime} f(y)$. Any such reducing map $f$ induces an injection $\tilde{f}: X / E \rightarrow X^{\prime} / E^{\prime}$ of the quotient spaces given by $\tilde{f}\left([x]_{E}\right)=[f(x)]_{E^{\prime}}$ where $[x]_{E}$ denotes the $E$-equivalence class of $x$. We also use $E \approx^{*} E^{\prime}: \Leftrightarrow E \leq E^{\prime} \wedge E^{\prime} \leq E$ for the bi-reducibility relation.

(ii) We say that $E$ is (Borel) embeddable in $E^{\prime}$, in symbols $E \sqsubseteq E^{\prime}$, if $E$ is reducible to $E^{\prime}$ by an injective Borel map. We also use $E \approx E^{\prime}: \Leftrightarrow E \sqsubseteq$ $E^{\prime} \wedge E^{\prime} \sqsubseteq E$ for the bi-embeddability relation.

If we denote by $E \cong E^{\prime}$ the relation of (Borel) isomorphism between $E$ and $E^{\prime}$, i.e. the existence of a Borel bijection $f: X \rightarrow X^{\prime}$ such that $x E y \Leftrightarrow$ $f(x) E^{\prime} f(y)$, then it is clear (since a Borel injective image of a Borel set is Borel) that

$$
E \sqsubseteq E^{\prime} \Leftrightarrow \exists \text { Borel } Y \subseteq X^{\prime}\left(E \cong E^{\prime} \uparrow Y\right) .
$$

(iii) Finally, we say that $E$ is (Borel) invariantly embeddable to $E^{\prime}$, in symbols $E \sqsubseteq^{i} E^{\prime}$, if $E \cong E^{\prime} \uparrow Y$, where $Y$ is a Borel subset of $X^{\prime}$ invariant under $E^{\prime}$ (i.e., $y \in Y, z E^{\prime} y \Rightarrow z \in Y$ ). By the usual Schroeder-Bernstein argument,

$$
E \cong E^{\prime} \Leftrightarrow E \sqsubseteq^{i} E^{\prime} \wedge E^{\prime} \sqsubseteq^{i} E .
$$

Our primary goal here is to study countable Borel equivalence relations, i.e., Borel equivalence relations $E$ for which every equivalence class $[x]_{E}$ is countable. 
Let $G$ be a countable group and $X$ a standard Borel space. A Borel action is an action $(g, x) \mapsto g \cdot x$ of $G$ on $X$ (i.e. a map from $G \times X$ into $X$ satisfying $1 \cdot x=x, g h \cdot x=g \cdot(h \cdot x))$ such that for each $g, g(x):=g \cdot x$ is Borel (thus a Borel automorphism of $X$ ). This is the same thing as saying that $(g, x) \mapsto g \cdot x$ is Borel from $G \times X$ into $X$, with $G$ given the discrete Borel structure (and the product has as usual the product Borel structure). Given a Borel action of $G$ on $X$, we denote by $E_{G}$ the induced equivalence relation

$$
x E_{G} y \Leftrightarrow \exists g \in G(y=g \cdot x) .
$$

This is clearly a countable Borel equivalence relation on $X$. Conversely we have

Theorem 1.1 (Feldman-Moore [FM]). Let $E$ be a countable Borel equivalence relation on a standard Borel space $X$. Then there are a countable group $G$ and a Borel action of $G$ on $X$ such that $E=E_{G}$.

We would like to mention first some consequences of this result.

Given any standard Borel space $X$ and a countable group $G$, denote by $X^{G}$ the set of maps from $G$ into $X$ with the usual product Borel structure. (If $\operatorname{card}(G)=n$, with $n \leq \aleph_{0}$, then $X^{G}$ is Borel isomorphic to $X^{n}$.) There is a canonical Borel action of $G$ on $X^{G}$ given by $g \cdot p(h)=p\left(g^{-1} h\right)$ for $p \in X^{G}$, $g \in G$. We denote by $E(G, X)$ the corresponding equivalence relation. This equivalence relation, for $X=2^{\mathrm{N}}$, is invariantly universal among all $E_{G}$ in the following sense.

Proposition 1.2. Let $G$ be a countable group and $E_{G}$ the equivalence relation induced by a Borel G-action on $X$. Then $E_{G} \complement^{i} E\left(G, 2^{\mathbb{N}}\right)$.

Proof. Let $\left\{U_{i}\right\}_{i \in \mathbb{N}}$ be a sequence of Borel sets in $X$ separating points. Define $f: X \rightarrow\left(2^{\mathbb{N}}\right)^{G}$ by

$$
f(x)(g)(i)=1 \Leftrightarrow g^{-1} \cdot x \in U_{i} .
$$

Then $f$ is injective. Moreover $g \cdot f(x)=f(g \cdot x)$ so $f$ maps $X$ onto an invariant Borel subset $Y$ of $\left(2^{\mathbb{N}}\right)^{G}$ and shows, in particular, that $E_{G} \cong E\left(G, 2^{\mathbb{N}}\right)$ । $Y$.

By taking $G=F_{\omega}$, the free group with countably infinitely many generators, we obtain

Proposition 1.3. Let $E$ be a countable Borel equivalence relation. Then $E \sqsubseteq^{i}$ $E\left(F_{\omega}, 2^{\mathbb{N}}\right)$.

Concerning the embeddability relation $\sqsubseteq$, one can obtain some tighter results. Let us note the following propositions.

Proposition 1.4. Suppose $G$ is a homomorphic image of $H$. Then $E(G, X) \sqsubseteq^{i}$ $E(H, X)$.

Proof. If $\pi: H \rightarrow G$ is an onto homomorphism, define $p \in X^{G} \mapsto p^{*} \in X^{H}$ by $p^{*}(h)=p(\pi h)$. Then $h \cdot p^{*}=(\pi h \cdot p)^{*}$, so this map shows that $E(G, X) \sqsubseteq^{i}$ $E(H, X)$.

Proposition 1.5. If $G \subseteq H$ (i.e., $G$ is a subgroup of $H$ ), then $E(G, X) \sqsubseteq$ $E(H, X)$. 
Proof. Define $p \in X^{G} \mapsto p^{*} \in X^{H}$ by

$$
p^{*}(h)= \begin{cases}p(h), & \text { if } h \in G, \\ x_{0}, & \text { if } h \notin G,\end{cases}
$$

where $x_{0}$ is some fixed element of $X$.

Proposition 1.6. $E\left(G, 2^{\mathbb{Z}-\{0\}}\right) \sqsubseteq E(G \times \mathbb{Z}, 3)$.

Proof. Define $p \in\left(2^{\mathbb{Z}-\{0\}}\right)^{G} \mapsto p^{*} \in 3^{G \times \mathbb{Z}}$ by

$$
p^{*}(g, n)= \begin{cases}p(g)(n), & \text { if } n \neq 0, \\ 2, & \text { if } n=0 .\end{cases}
$$

Then $q=g \cdot p \Rightarrow q^{*}=(g, 0) \cdot p^{*}$. Conversely if $q^{*}=(g, n) \cdot p^{*}$ and $n=0$ then $q=g \cdot p$. If $n \neq 0$, then $q^{*}\left(g_{0}, n_{0}\right)=p^{*}\left(g^{-1} g_{0}, n_{0}-n\right)$, so $q\left(g_{0}\right)(n)=q^{*}\left(g_{0}, n\right)=p^{*}\left(g^{-1} g_{0}, 0\right)=2$, a contradiction.

Proposition 1.7. $E(G, 3) \sqsubseteq E\left(G \times \mathbb{Z}_{2}, 2\right)$.

Proof. View 0 as encoded by 00,1 by 01 , and 2 by 11 . Define $p \in 3^{G} \mapsto p^{*} \in$ $2^{G \times \mathbb{Z}_{2}}$ by

$$
\begin{aligned}
p^{*}(g, i)=0, & \text { if } p(g)=0, \\
& =0, \quad \text { if } p(g)=1, i=0, \\
& =1, \quad \text { if } p(g)=1, i=1, \\
& =1, \quad \text { if } p(g)=2 .
\end{aligned}
$$

If $q=g \cdot p$, then $q^{*}=(g, 0) \cdot p^{*}$. Conversely, if $q^{*}=(g, i) \cdot p^{*}$ and $i=0$, then $q=g \cdot p$. If $i=1$ and $q\left(g_{0}\right)=1$ for some $g_{0}$, then $q^{*}\left(g_{0}, 0\right)=0, q^{*}\left(g_{0}, 1\right)=$ 1 but $q^{*}\left(g_{0}, i\right)=p^{*}\left(g^{-1} g_{0}, 1+i\right)$, thus $p^{*}\left(g^{-1} g_{0}, 0\right)=q^{*}\left(g_{0}, 1\right)=1$, so $p\left(g^{-1} g_{0}\right)=2$, while $p^{*}\left(g^{-1} g_{0}, 1\right)=q^{*}\left(g_{0}, 0\right)=0$, a contradiction. So we must have that $q \in\{0,2\}^{G}$ in which case it is easy to see that $q=g \cdot p$.

Letting $F_{n}:=$ the free group with $n$ generators, we have now from the preceding results that $E\left(F_{2}, 2\right)$ is universal among all countable Borel $E$ in the following sense.

Proposition 1.8. Let $E$ be a countable Borel equivalence relation. Then $E \sqsubseteq$ $E\left(F_{2}, 2\right)$.

Proof. We have

$$
\begin{aligned}
E & \sqsubseteq E\left(F_{\omega}, 2^{\mathbb{N}}\right), \quad \text { by Proposition } 1.3 \\
& \cong E\left(F_{\omega}, 2^{\mathbb{Z}-\{0\}}\right) \\
& \sqsubseteq E\left(F_{2}, 2^{\mathbb{Z}-\{0\}}\right), \quad \text { by Proposition } 1.5 \\
& \sqsubseteq E\left(F_{2} \times \mathbb{Z}, 3\right), \quad \text { by Proposition } 1.6 \\
& \sqsubseteq E\left(F_{2} \times \mathbb{Z} \times \mathbb{Z}_{2}, 2\right), \quad \text { by Proposition } 1.7 \\
& \sqsubseteq E\left(F_{\omega}, 2\right), \quad \text { by Proposition } 1.4 \\
& \sqsubseteq E\left(F_{2}, 2\right), \quad \text { by Proposition } 1.5 .
\end{aligned}
$$


We do not know whether every countable Borel $E$ is of the form $E_{F_{2}}$, or, even more, whether $E \sqsubseteq^{i} E\left(F_{2}, 2\right)$ (see however Proposition 2.4 for an affirmative answer in a special case).

\section{COMPRESSIBILITY}

Given a countable Borel equivalence relation $E$ on $X$, we denote by $[[E]]$ the set of Borel bijections $f: A \rightarrow B$ with $A, B$ Borel subsets of $X$, which have the property that $\forall x \in A(f(x) E x)$. Note that $f: A \rightarrow B$ belongs to [[E]] iff, for any countable group $G$ with $E=E_{G}$, there are Borel partitions $A=\bigcup_{i \in \mathbb{N}} A_{i}, B=\bigcup_{i \in \mathbb{N}} B_{i}$ and $g_{i} \in G$ with $g_{i}\left[A_{i}\right]=B_{i}$ and $g_{i}=f$ on $A_{i}$.

For Borel sets $A, B \subseteq X$ we let now $A \sim B: \Leftrightarrow \exists f \in[[E]](f: A \rightarrow B)$. This means that for any $E$-equivalence class $C$, if $A^{C}=A \cap C, B^{C}=B \cap C$, there is a 1-1 correspondence of $A^{C}$ with $B^{C}$ depending in a "uniform Borel" way on $C$. Let also

$$
A \preceq B: \Leftrightarrow \exists \text { Borel } B^{\prime} \subseteq B\left(A \sim B^{\prime}\right) .
$$

Note that by the usual Schroeder-Bernstein argument

$$
A \sim B \Leftrightarrow A \preceq B \wedge B \preceq A .
$$

We discuss now the notion of compressibility of an equivalence relation arising in the measure theoretic aspects of this subject in the Hopf Theorem (see e.g. Weiss [W1] or Friedman [Fr]) and studied extensively in the Borel context in Nadkarni [N1, N2], Chaube-Nadkarni [CN1, CN2].

Let $E$ be a countable Borel equivalence relation on $X$. We call $E$ compressible if there is Borel $A \subseteq X$ with $X \sim A$ such that $X \backslash A$ is full (or a complete section), i.e., meets every $E$-equivalence class. This just means that each $E$-equivalence class can be mapped into a proper subset of itself in a uniform Borel way, i.e., every equivalence class is Dedekind infinite in a uniform Borel way. We have the following useful reformulations of compressibility.

Proposition 2.1. Let $E$ be a countable Borel equivalence relation on $X$. Then the following are equivalent:

(1) $E$ is compressible.

(2) There is a pairwise disjoint sequence $\left\{A_{n}\right\}$ of full Borel sets with $A_{n} \sim A_{m}$ for all $n, m$.

(3) $X$ is E-paradoxical, where a Borel subset $A \subseteq X$ is E-paradoxical if there are disjoint Borel $B, C \subseteq A$ with $A \sim B$ and $A \sim C$.

Proof. (1) $\Rightarrow(2)$. Let $f: X \rightarrow B, f \in[[E]]$ with $X \backslash B=A$ full. Put $A_{n}=$ $f^{n}[A]$.

$(2) \Rightarrow(3)$. Let $f_{n}: A_{0} \rightarrow A_{n}, f_{n} \in[[E]]$ and let $E=E_{G}$, where $G=\left\{g_{n}\right\}$ is a countable group. Define $N: X \rightarrow \mathbb{N}$ by

$$
N(x)=\text { least } n \text { such that } g_{n} \cdot x \in A_{0}
$$

(this exists since $A_{0}$ is full). Clearly $N$ is Borel. Define now $h_{0}, h_{1}$ on $X$ by

$$
h_{0}(x)=f_{2 N(x)}\left(g_{N(x)} \cdot x\right), \quad h_{1}(x)=f_{2 N(x)+1}\left(g_{N(x)} \cdot x\right) .
$$

If $h_{0}[X]=B, h_{1}[X]=C$ then clearly $h_{0}: X \rightarrow B, h_{1}: X \rightarrow C$ are in [[E]] and so $X \sim B, X \sim C$. But also $B \cap C=\varnothing$ and we are done.

(3) $\Rightarrow(1)$. If $f: X \rightarrow B$ is in [[E]] and $g: X \rightarrow C$ is in [[E]] with $B \cap C=\varnothing$, then clearly $C$ and thus $X \backslash B$ is full, so $E$ is compressible. 
We call now a Borel subset $A \subseteq X$ compressible if $E \backslash A$ is compressible. A well-known basic fact (see [N1, 5.7]) about compressible sets is the following.

Proposition 2.2. Let $E$ be a countable Borel equivalence relation on $X$ and $A \subseteq X$ a Borel set. If $A$ is compressible, then $A \sim[A]_{E}$ where

$$
[A]_{E}=\{x \in X: \exists y[y \in A \wedge x E y]\}
$$

is the E-saturation of $A$. In particular, $[A]_{E}$ is also compressible.

Proof. By Schroeder-Bernstein it is enough to show $[A]_{E} \preceq A$. By 2.1, let $\left\{A_{n}\right\}$ be pairwise disjoint Borel subsets of $A$ full for $E\left\lceil A\right.$ and thus for $E\left\lceil[A]_{E}\right.$, with $A_{n} \sim A_{m}$. Then exactly as in the proof of $(2) \Rightarrow(3)$ of 2.1 (applied with $\left.X=[A]_{E}\right)$, we have that $[A]_{E} \preceq \bigcup_{n} A_{n} \subseteq A$.

This has the following immediate corollary.

Proposition 2.3. Let $E, F$ be countable Borel equivalence relations. Then, if $E$ is compressible and $E \sqsubseteq F$, we have $E \sqsubseteq^{i} F$. In particular, for compressible $E, F: E \approx F \Leftrightarrow E \cong F$.

Proof. Let $E \sqsubseteq F$, so that $E \cong F \mid A, A$ a Borel set. Then $A$ is compressible (for $F$ ). So $A \sim[A]_{F}$, thus $E \cong F|A \cong F|[A]_{F}$, i.e., $E \sqsubseteq^{i} F$.

Another corollary is the following.

Proposition 2.4. Let $E$ be a countable Borel equivalence relation. If $E$ is compressible, then $E \bigsqcup^{i} E\left(F_{2}, 2\right)$ and so in particular $E$ is inauced by an action of $F_{2}$.

We will provide now some further equivalents of compressibility. For each set $\Omega$, let $I(\Omega):=\Omega \times \Omega$ be the largest equivalence relation on $\Omega$. Put $I_{\infty}:=I(\mathbb{N})$. For equivalence relations $E$ on $X$ and $F$ on $Y, E \times F$ denotes the product (on $X \times Y$ ) where

$$
(x, y) E \times F\left(x^{\prime}, y^{\prime}\right): \Leftrightarrow x E x^{\prime} \wedge y F y^{\prime} .
$$

Call an equivalence relation $E$ aperiodic if every $E$-equivalence class is infinite. Finally, call a Borel equivalence relation $E$ on $X$ smooth if there is a Borel function $f: X \rightarrow Y$ ( $Y$ some standard Borel space) with $x E y \Leftrightarrow f(x)=f(y)$, i.e. $E \leq \Delta(Y)$, where $\Delta(\Omega):=$ the equality on $\Omega$. For countable Borel $E$, this is equivalent to the existence of a Borel selector, i.e., a Borel function $S: X \rightarrow X$ with $x E y \Rightarrow S(x)=S(y), S(x) E x$.

As usual, $E \subseteq F$ means that $E$ is a subequivalence relation of $F$ (i.e. $x E y \Rightarrow x F y)$.

Proposition 2.5. Let $E$ be a countable Borel equivalence relation. Then the following are equivalent:

(1) $E$ is compressible,

(2) $E \cong E \times I_{\infty}$,

(3) there is a smooth aperiodic Borel equivalence relation $F \subseteq E$.

Proof. (1) $\Rightarrow(2)$. Clearly $E \cong\left(E \times I_{\infty}\right) \backslash(X \times\{0\})$ and so $\left.E \cong\left(E \times I_{\infty}\right)\right\rceil$ $[X \times\{0\}]_{E \times I_{\infty}}=E \times I_{\infty}$, by 2.3 .

$(2) \Rightarrow(3)$. It is enough to show that (3) holds for $E \times I_{\infty}$. Define $F \subseteq E \times I_{\infty}$ by $(x, n) F(y, m) \Leftrightarrow x=y$. 
(3) $\Rightarrow(1)$. Note that, since $F$ admits a Borel selector and each $F$-equivalence class is infinite, $F$ is compressible. But it is also straightforward to check that if $F$ is compressible and $F \subseteq E$, then $E$ is compressible.

Note that in $(3) \Rightarrow(1)$, one only uses the following: $F \subseteq E, F$ is smooth Borel and every $E$-equivalence class contains an infinite $F$-equivalence class.

For each set $\Omega, E_{0}(\Omega)$ is the equivalence relation on $\Omega^{\mathbb{N}}$ given by

$$
x E_{0}(\Omega) y: \Leftrightarrow \exists n \forall m \geq n\left(x_{m}=y_{m}\right) .
$$

We let $E_{0}:=E_{0}(2)$. We also denote by $E_{t}(\Omega)$ the equivalence relation on $\Omega^{\mathbb{N}}$ given by

and let again $E_{t}:=E_{t}(2)$.

$$
x E_{t}(\Omega) y: \Leftrightarrow \exists n \exists m \forall k\left(x_{n+k}=y_{m+k}\right)
$$

Consider then the following examples of compressible $E$ :

(a) Let $X=\mathbb{N}^{\mathbb{N}}$ and $E=E_{0}(\mathbb{N})$. To see that $E$ is compressible, note that $F \subseteq E$, where $x F y: \Leftrightarrow x^{\prime}=y^{\prime}$, with $x^{\prime}=\left(x_{1}, x_{2}, \ldots\right)$ if $x=$ $\left(x_{0}, x_{1}, x_{2}, \ldots\right)$.

(b) Let $X=2^{\mathbb{N}}$ and $E=E_{t}$. Again $E$ is compressible since $F \subseteq E$, where $x F y: \Leftrightarrow[x, y$ are eventually equal to 1$] \vee\left[x=1^{n \wedge} 0^{\wedge} x^{\prime} \wedge y=1^{m \wedge} 0^{\wedge} y^{\prime}\right.$ with $\left.x^{\prime}=y^{\prime}\right]$.

We can also use the present ideas to provide some alternative characterizations of the notion of bi-reducibility $E \approx^{*} F$ introduced in $\S 1$.

We call two Borel equivalence relations $E, F$ stably isomorphic, in symbols $E \cong_{s} F$ if there are Borel sets $A, B$ full for $E, F$ resp., such that $E \mid A \cong$ $F \mid B$.

We then have

Proposition 2.6. Let $E, F$ be countable Borel equivalence relations on $X, Y$ resp. Then the following are equivalent:

(1) $E \approx^{*} F$,

(2) $E \cong_{s} F$,

(3) $E \times I_{\infty} \cong F \times I_{\infty}$.

Proof. (3) $\Rightarrow$ (2). Let $f: X \times \mathbb{N} \rightarrow Y \times \mathbb{N}$ be a Borel isomorphism of $E \times$ $I_{\infty}, F \times I_{\infty}$. Put $A=\{x \in X: \exists n \exists y(f(x, n)=(y, 0))\}$. For $x \in A$, let $N(x)=$ least $n$ with $\exists y[f(x, n)=(y, 0)]$. Put $g(x)=$ the unique $y$ with $f(x, N(x))=(y, 0)$. Clearly $g$ is Borel injective. Let $g[A]=B$. Clearly $A, B$ are full and $g$ shows that $E\lceil A \cong F \mid B$.

(2) $\Rightarrow(1)$. Let $A, B$ be Borel full with $E\left\lceil A \cong F \mid B\right.$ via $f$. Let $E=E_{G}$ with $G=\left\{g_{n}\right\}$ a countable group. Define for each $x \in X, N(x)=$ least $n$ with $g_{n} \cdot x \in A$ and $g: X \rightarrow Y$ by $g(x)=f\left(g_{N(x)} \cdot x\right)$. Then $g$ is Borel and reduces $E$ to $F$, thus $E \leq F$. Similarly $F \leq E$, so $E \approx^{*} F$.

(1) $\Rightarrow(3)$. Suppose $f: X \rightarrow Y$ reduces $E$ to $F$ and $g: Y \rightarrow X$ reduces $F$ to $E$. Let $\tilde{f}: X / E \rightarrow Y / F$ be the induced injection given by $\tilde{f}\left([x]_{E}\right)=[f(x)]_{F}$ and similarly for $\tilde{g}: Y / F \rightarrow X / E$. By Schroeder-Bernstein applied to $\tilde{f}, \tilde{g}$, we can partition $X$ into $E$-invariant Borel sets $A, B$ and $Y$ into $F$-invariant Borel sets $C, D$ such that $\tilde{f}$ maps $A / E$ onto $C / F$ and $\tilde{g}$ maps $D / F$ onto $B / E$. Therefore $f[A]$ is full in $C$ and $g[D]$ is full in $B$. Note that since $f, g$ are countable-to- $1, f[A], g[D]$ are Borel sets.

We will show that $\left(E \times I_{\infty}\right) \uparrow(B \times \mathbb{N}) \cong\left(F \times I_{\infty}\right) \backslash D^{\prime}$, where $D^{\prime}$ is Borel full in $D \times \mathbb{N}$, and $\left(F \times I_{\infty}\right)\left|(C \times \mathbb{N}) \cong\left(E \times I_{\infty}\right)\right| A^{\prime}$, where $A^{\prime}$ is Borel 
full in $A \times \mathbb{N}$. Since $E \times I_{\infty}, F \times I_{\infty}$ are compressible, this will imply that $\left.\left(E \times I_{\infty}\right)\right\rceil(B \times \mathbb{N}) \cong\left(F \times I_{\infty}\right)\left\lceil(D \times \mathbb{N})\right.$ and $\left(F \times I_{\infty}\right)\left\lceil(C \times \mathbb{N}) \cong\left(E \times I_{\infty}\right)\right\rceil$ $(A \times \mathbb{N})$, so $E \times I_{\infty} \cong F \times I_{\infty}$.

Let us work with $\left(F \times I_{\infty}\right) \backslash(C \times \mathbb{N})$, the other case being similar. Let $\left(F \times I_{\infty}\right)=E_{H}$, with $H=\left\{h_{n}\right\}$ a countable group. Given $z \in C \times \mathbb{N}$, let $N(z)$ be the least $n$ with $h_{n} \cdot z=(y, 0)$ and $y \in f[A]$. Put $h_{N(z)} \cdot z=(p(z), 0)$, $p$ Borel. Let $f^{*}: f[A] \rightarrow A$ be Borel with $f\left(f^{*}(y)\right)=y$, which exists since $f$ is countable-to- 1 . Finally put $q(z)=\left(f^{*}(p(z)), N(z)\right)$. Then $q$ is a Borel injection, $A^{\prime}=q[C \times \mathbb{N}]$ is full in $A \times \mathbb{N}$, and $q$ shows that $\left(F \times I_{\infty}\right) \uparrow(C \times \mathbb{N}) \cong$ $\left(E \times I_{\infty}\right)\left\lceil A^{\prime}\right.$.

We close this section with the following question:

Let $E, F$ be aperiodic countable Borel equivalence relations. If $E \approx^{*} F$, is it true that $E \approx F$ ?

Note that this is equivalent to asking whether $E \times I_{\infty} \sqsubseteq E$ for all aperiodic $E$. A counterexample could be provided, for example, by finding a property $\mathscr{P}(E)$ of aperiodic countable Borel equivalence relations, which is preserved under restriction (i.e. $\mathscr{P}(E) \Rightarrow \mathscr{P}(E\lceil A)$ ) but not extension from a full subset (i.e., $\mathscr{P}(E\lceil A)$ does not necessarily imply $\mathscr{P}(E)$, whenever $A$ is full for $E)$.

\section{INVARIANT AND QUASI-INVARIANT MEASURES}

Let $X$ be a standard Borel space. By a measure on $X$ we will always mean a $\sigma$-finite Borel measure on $X$. If $\mu$ is a measure on $X$ and $\mu(X)<\infty$ we call $\mu$ finite; if $\mu(X)=1, \mu$ is a probability measure. For any Borel $f: X \rightarrow Y$, we define $f \mu$ to be the measure $f \mu(A)=\mu\left(f^{-1}[A]\right)$.

For any measures $\mu, \nu$ on $X, \mu<<\nu$ means that $\mu$ is absolutely continuous to $\nu$ (i.e., $\nu(A)=0 \Rightarrow \mu(A)=0$ ), and we let

$$
\mu \sim \nu: \Leftrightarrow \mu<<\nu \wedge \nu<<\mu .
$$

This is an equivalence relation on measures, whose equivalence classes $[\mu]_{\sim}$ (or just $[\mu]$ ) are called measure classes. Note that for any $\mu$ there is a probability measure $\nu$ with $\nu \in[\mu]$. A measure $\mu$ is orthogonal to $\nu$, in symbols $\mu \perp \nu$, if there is a Borel partition $X=A \cup B$ with $\mu(A)=\nu(B)=0$.

Now let $G$ be a countable group acting in a Borel way on $X$. A measure $\mu$ is called $G$-invariant if $g \cdot \mu=\mu$ (here $g \cdot \mu$ is the measure $g \mu$ for $g(x)=g \cdot x$ so that $\left.g \cdot \mu(A)=\mu\left(g^{-1} \cdot A\right)\right)$ and $G$-quasi-invariant if $g \cdot \mu \sim \mu$.

Suppose now $E$ is a countable Borel equivalence relation on $X$ and $\mu$ a measure on $X$. We call $\mu E$-invariant if $\mu$ is $G$-invariant for any $G$ acting in a Borel way on $X$ with $E=E_{G}$. It is easy to see that this property is independent of $G$ and is equivalent to either of the properties below:

(1) If $f \in[[E]], f: A \rightarrow B$, then $\mu(A)=\mu(B)$.

(2) If $f \in[[E]], f: X \rightarrow X$, then $f \mu=\mu$.

With the preceding notation, we call $\mu E$-quasi-invariant, if for any $G$ with $E=E_{G}, \mu$ is $G$-quasi-invariant. Again it is easy to see that this is independent of $G$ and equivalent to the following properties:

(1) For any Borel $A \subseteq X$, if $\mu(A)=0$ then $\mu\left([A]_{E}\right)=0$.

(2) If $f \in[[E]], f: A \rightarrow B$ and $\mu(A)=0$, then $\mu(B)=0$.

A measure $\mu$ on $X$ is caled $E$-ergodic if, for any Borel invariant $A, \mu(A)=$ 0 or $\mu(X \backslash A)=0$. It is called E-nonatomic if $\mu\left([x]_{E}\right)=0$ for each $E$ equivalence class $[x]_{E}$, i.e., $\mu(\{x\})=0$ for each point $x$. 
Let us notice first a few simple facts:

Proposition 3.1. Let $E$ be a countable Borel equivalence relation and $\mu$ a measure. Then there is an E-quasi-invariant measure $\mu^{*}$ such that:

(1) If $A$ is Borel E-invariant, then $\mu(A)=\mu^{*}(A)$.

(2) If $\mu$ is E-ergodic, so is $\mu^{*}$.

(3) $\mu^{*}$ is a least, in the sense of $<<$, measure such that $\mu^{*}$ is $E$-quasiinvariant and $\mu<<\mu^{*}$.

Proof. Let $E=E_{G}$ with $G=\left\{g_{1}, g_{2}, \ldots\right\}$. First, we claim that we can write $X$ (the space on which $E$ lives) as $X=\bigcup_{n} A_{n}$, with $A_{n}$ Borel, such that for all $i, \mu\left(g_{i} \cdot A_{n}\right)<\infty$. To prove this, call a Borel set $A \subseteq X$ nice if $\mu\left([A]_{E}\right)>0$ and $\mu\left(g_{i} \cdot A\right)<\infty$, for all $i$. We claim that nice sets exist: Indeed, fix a Borel set $B$ with $\infty>\mu(B)>0$. Then write $g_{i} \cdot B=\bigcup_{n} B_{i, n}$, where the sets $B_{i, n}$ are Borel, increasing with $n$ and $\mu\left(B_{i, n}\right)<\infty$. For each $i$, fix $n=n(i)$ such that $\mu\left(g_{i}^{-1} \cdot B_{i, n}\right)>\mu(B)-\mu(B) / 2^{i+2}$. Put $A=\bigcap_{i} g_{i}^{-1} \cdot B_{i, n(i)}$. Then $\mu(A)>0$, so $\mu\left([A]_{E}\right)>0$, and $g_{i} \cdot A \subseteq B_{i, n(i)}$, so $\mu\left(g_{i} \cdot A\right)<\infty$. By a simple exhaustion argument now, we can find a sequence $C_{i}$ of nice Borel sets with $\bigcup_{i}\left[C_{i}\right]=X$. Let $\left\{A_{n}\right\}=\left\{g_{j} \cdot C_{i}\right\}$.

Fix now such a sequence $\left\{A_{n}\right\}$ and choose a sequence $\left\{\alpha_{i}\right\}$ of positive reals such that $\alpha=\sum \alpha_{i}<\infty$ and $\sum \alpha_{i} \mu\left(g_{i} \cdot A_{n}\right)<\infty$, for all $n$. Define then $\mu^{*}$ by

$$
\mu^{*}(A)=\alpha^{-1} \sum \alpha_{i} \mu\left(g_{i} \cdot A\right) .
$$

Clearly $\mu<<\mu^{*}$ and (1), (2) hold. For (3), assume $\mu<<\nu$ and $\nu$ is $E$ quasi-invariant. We will show that $\mu^{*}<<\nu$ : For any Borel $A \subseteq X, \nu(A)=$ $0 \Rightarrow \forall g \in G(\nu(g \cdot A)=0) \Rightarrow \forall g \in G(\mu(g \cdot A)=0) \Rightarrow \mu^{*}(A)=0$.

Proposition 3.2. Let $E$ be a countable Borel equivalence relation and $A \subseteq X$ a full Borel set. Let $\mu$ be a measure on $A$ which is $E \mid A$-invariant. Then there is a unique E-invariant measure $\nu$ on $X$ with $\nu(B)=\mu(B)$ for all Borel sets $B \subseteq A$. If $\mu$ is nonatomic or ergodic (for $E\lceil A)$, so is $\nu$ (for $E$ ).

(In general, $\nu$ will not be finite even if $\mu$ is.)

Proof. Let $E=E_{G}, G=\left\{g_{0}, g_{1}, \ldots\right\}$ a countable group, with $g_{0}=1$. Put $A_{i}=g_{i} \cdot A, B_{i}=A_{i} \backslash \bigcup_{j<i} A_{j}$, so that $\left\{B_{i}\right\}$ is a Borel partition of $X$. Put

$$
\nu(B)=\sum_{i=0}^{\infty} \mu\left(g_{i}^{-1} \cdot\left(B \cap B_{i}\right)\right) .
$$

Clearly $\nu(B)=\mu(B)$, for $B \subseteq A \quad\left(=A_{0}=B_{0}\right)$.

To see that $\nu$ is $E$-invariant, note that, if for Borel $B \subseteq X, g \in G$ we define

$$
B_{i, k}=\left\{x \in B \cap B_{i}: g \cdot x \in B_{k}\right\},
$$

then $\left\{B_{i, k}\right\}$ is a Borel partition of $B$. So to show $\nu(B)=\nu(g \cdot B)$, it is enough to show that $\nu\left(B_{i, k}\right)=\nu\left(g \cdot B_{i, k}\right)$. Equivalently, assume that $B \subseteq B_{i}$ and $g \cdot B \subseteq B_{k}$. Now $\nu(B)=\mu\left(g_{i}^{-1} \cdot B\right)$ and $\nu(g \cdot B)=\mu\left(g_{k}^{-1} g \cdot B\right)$. Define $h: g_{i}^{-1} \cdot B \rightarrow g_{k}^{-1} g \cdot B$ by $h=g_{k}^{-1} g g_{i}\left\lceil g_{i}^{-1} \cdot B\right.$, so that $h \in[[E \backslash A]]$. Thus by the $E\left\lceil A\right.$-invariance of $\mu$ we have $\mu\left(g_{i}^{-1} \cdot B\right)=\mu\left(g_{k}^{-1} g \cdot B\right)$ and we are done. 
Assume now $\mu$ is nonatomic. Then clearly so is $\nu$. If $\mu$ is ergodic, let $B \subseteq X$ be $E$-invariant. Then $A \cap B$ is $E\lceil A$-invariant. Say $\mu(A \cap B)=0$. Then $\nu(A \cap B)=0$, so $\nu\left([A \cap B]_{E}\right)=\nu(B)=0$. Similarly if $\mu(A \backslash B)=0$.

Proposition 3.3. Let $E$ be a countable Borel equivalence relation and $A \subseteq X$ a full Borel set. Let $\mu$ be a probability measure on $A$ which is $E$ \A-quasiinvariant. Then there is a probability measure $\nu$ on $X$ such that $\nu$ is $E$-quasiinvariant and $\nu(B)=\nu(A) \cdot \mu(B)$ for all Borel sets $B \subseteq A$. If $\mu$ is nonatomic or ergodic (for $E\lceil A)$, so is $\nu$ (for $E$ ).

Proof. Let $E=E_{G}, G=\left\{g_{i}\right\}, g_{0}=1$. Define $A_{i}, B_{i}$ as in 3.2 and put

$$
\nu(B)=c_{\mu} \sum_{i=0}^{\infty} 2^{-i-1} \mu\left(g_{i}^{-1} \cdot\left(B \cap B_{i}\right)\right)
$$

for $B \subseteq X$ Borel, where $c_{\mu}$ is a positive constant such that $\nu(X)=1$. It is easy to check that $\nu(B)=\nu(A) \cdot \mu(B)$, for $B \subseteq A$.

We will check next that $\nu$ is $E$-quasi-invariant. Let $B \subseteq X$ be Borel with $\nu(B)=0$; we will show that $\nu\left([B]_{E}\right)=0$. We can assume that $B \subseteq B_{i}$ for some $i$. Since $[B]_{E}=\left[g_{i}^{-1} \cdot B\right]_{E}$, it suffices to show that

$$
C \subseteq A \text { Borel } \wedge \mu(C)=0 \Rightarrow \nu\left([C]_{E}\right)=0 .
$$

For that it is enough again to show that $\mu\left(g_{i}^{-1} \cdot\left([C]_{E} \cap B_{j}\right)\right)=0$ for each $j$. But $g_{j}^{-1} \cdot\left([C]_{E} \cap B_{j}\right) \subseteq[C]_{E\lceil A}$, and $\mu\left([C]_{E \nmid A}\right)=0$ by the $E\lceil A$-quasi-invariance of $\mu$.

If $\mu$ is nonatomic, clearly so is $\nu$. If $\mu$ is $E \uparrow A$-ergodic, then $\nu$ is $E$ ergodic as in 3.2 .

In particular (from 3.2), if $E \sqsubseteq F$ and $E$ has an invariant (nonatomic, ergodic) measure, then so does $F$.

We now have the following result which is a special case of the theorem in Harrington-Kechris-Louveau [HKL], but was already proved earlier in Effros [E1, E2] and Weiss [W2].

Theorem 3.4 (Effros [E1, E2], Weiss [W2]). Let $E$ be a countable Borel equivalence relation. Then the following are equivalent:

(1) $E$ is not smooth;

(2) $E_{0} \sqsubseteq E$;

(3) E admits a nonatomic, ergodic, (quasi-)invariant measure.

Since it is easy to check that every smooth countable Borel $E$ on an uncountable space $X$ admits a nonatomic invariant measure, it follows that every countable Borel equivalence relation $E$ on an uncountable space $X$ admits a nonatomic invariant measure.

Not every countable Borel equivalence relation admits a finite invariant measure. It is clear that if $E$ is compressible it cannot admit such a measure. The following basic result of Nadkarni shows that this is the only obstruction and provides a fundamental relation between compressibility and existence of finite invariant measures.

Theorem 3.5 (Nadkarni [N2]). Let $E$ be a countable Borel equivalence relation. Then the following are equivalent: 
(1) $E$ is not compressible,

(2) E admits an invariant probability measure.

(In [N2], this is stated and proved only for hyperfinite $E$ (see $\S 5$ ), but the proof can be easily generalized to arbitrary countable $E$.)

\section{$\S 4$. THE SPACES OF INVARIANT AND QUASI-INVARIANT MEASURES}

Let $X$ be a standard Borel space. Denote by $\mathscr{M}(X)$ the space of probability measures on $X$ equipped with the Borel structure generated by the maps $\mu \mapsto$ $\mu(A)$, where $A \subset X$ is Borel. This is a standard Borel space (see, e.g., [Va]). If now $E$ is a countable Borel equivalence relation on $X$, put

$$
\begin{aligned}
& \mathscr{I}_{0}(E):=\{\mu \in \mathscr{M}(X): \mu \text { is } E \text {-invariant and nonatomic }\}, \\
& \mathscr{E}_{0}(E):=\left\{\mu \in \mathscr{I}_{0}(E): \mu \text { is } E \text {-ergodic }\right\} .
\end{aligned}
$$

Then $\mathscr{I}_{0}(E), \mathscr{E}_{0}(E)$ are Borel subsets of $\mathscr{M}(X)$ (see again [Va]). Let also

$$
\begin{aligned}
& \mathscr{Q S}_{0}(E):=\{\mu \in \mathscr{M}(X): \mu \text { is } E \text {-quasi-invariant and nonatomic }\}, \\
& \mathscr{Q E}_{0}(E):=\left\{\mu \in \mathscr{Q}_{0}(E): \mu \text { is } E \text {-ergodic }\right\} .
\end{aligned}
$$

Again $\mathscr{Q S}_{0}(E)$ is Borel, a fact which can be seen as follows: Since any two uncountable Borel spaces are Borel isomorphic, we can assume that $X=2^{\mathrm{N}}$. Let $\left\{C_{n}\right\}$ be an enumeration of the clopen subsets of $2^{\mathbb{N}}$. Then if $E=E_{G}$, with $G=\left\{g_{0}, g_{1}, \ldots\right\}$, we have that a probability measure $\mu$ on $2^{\mathbb{N}}$ is in $\mathscr{Q S}_{0}(E)$ iff (1) $\forall i \forall n \exists m \forall j\left[\mu\left(C_{j}\right)<1 /(m+1) \Rightarrow \mu\left(g_{i} \cdot C_{j}\right)<1 /(n+1)\right]$ and (2) $\forall n \exists j_{1} \cdots j_{k}\left[\left\{C_{j_{1}}, \ldots, C_{j_{k}}\right\}\right.$ is a partition of $2^{\mathbb{N}}$ and $\mu\left(C_{j_{p}}\right)<1 /(n+1)$, $1 \leq p \leq k]$. Although we do not need it, it can be shown that $\mathscr{Q E}_{0}(E)$ is also Borel. (This has been proved by A. Ditzen, using the result in [KP] and the method of [Va, Theorem 4.1].)

We will discuss $\mathscr{I}_{0}, \mathscr{E}_{0}$ (for hyperfinite $E$ ) in $\S 9$. Here we want to say a few things about $\mathscr{Q F}_{0}, \mathscr{Q E}_{0}$. Clearly both of these are $\sim$-invariant (where $\sim$ is equivalence of measures). For nonsmooth $E, \sim$ on $\mathscr{Q E}_{0}(E)$ is quite complicated. The fact below strengthens results in Krieger [Kr2] and Katznelson-Weiss $[\mathrm{KW}$.

Proposition 4.1. Let $E$ be a nonsmooth countable Borel equivalence relation. Then there is a Borel injection $F: 2^{\mathbb{N}} \rightarrow \mathscr{M}(X)$ with $\operatorname{range}(F) \subseteq \mathscr{Q E}_{0}(E)$ and $x E_{0} y \Leftrightarrow F(x) \sim F(y)$.

Proof. Since $E$ is not smooth, we have by 3.4 that there is a Borel injection $f: 2^{\mathbb{N}} \rightarrow X$ with $x E_{0} y \Leftrightarrow f(x) E f(y)$.

For each $\mu \in \mathscr{M}\left(2^{\mathbb{N}}\right)$, let $f \mu \in \mathscr{M}(X)$ be its image under $f$. So $f \mu$ is a measure on $f\left[2^{\mathbb{N}}\right]=A$. Applying 3.3 to $f \mu$ (with $X=[A]_{E}$ there), we can define an injective Borel map $g: \mathscr{Q S}_{0}\left(E_{0}\right) \rightarrow \mathscr{Q S}_{0}(E)$ such that $g\left[\mathscr{Q} \mathscr{E}_{0}\left(E_{0}\right)\right] \subseteq$ $\mathscr{Q E}_{0}(E)$ and $\mu \sim \nu \Leftrightarrow g(\mu) \sim g(\nu)$. So it is enough to show that there is a Borel injection $h: 2^{\mathbb{N}} \rightarrow \mathscr{M}\left(2^{\mathbb{N}}\right)$ with range $(h) \subseteq \mathscr{Q}_{0}\left(E_{0}\right)$ and $x E_{0} y \Leftrightarrow h(x) \sim h(y)$. This follows easily by a standard argument applying a result of Kakutani (see, e.g., [HS, 22.38]). For each $x \in 2^{\mathbb{N}}$, let $\mu_{x}$ be the product measure on $2^{\mathbb{N}}$, where for $x(n)=0$ the $n$th coordinate is given the $(1 / 2,1 / 2)$ measure, while for $x(n)=1$ it is given the $(3 / 4,1 / 4)$ measure. Then $x E_{0} y \Leftrightarrow \mu_{x} \sim \mu_{y}$. It is easy to check that $\mu_{x}$ is $E_{0}$-quasi-invariant and is well known that it is $E_{0}$-ergodic (see, e.g., [GM, 6.4.6]). Put $h(x)=\mu_{x}$. 
Finally we verify that the structure of $\mathscr{Q}_{0}(E), \mathscr{Q}_{0}(E)$ modulo $\sim$ depends only on the stable isomorphism type of $E$ (recall $\cong_{s}$ and 2.6 here).

Proposition 4.2. Let $E, F$ be countable Borel equivalence relations on the spaces $X, Y$ resp. If $E \cong_{s} F$, then there are Borel maps $\Phi: \mathscr{Q S}_{0}(E) \rightarrow \mathscr{Q S}_{0}(F)$ and $\Psi: \mathscr{Q S}_{0}(F) \rightarrow \mathscr{Q} \mathcal{S}_{0}(E)$ such that $\mu<<\nu \Leftrightarrow \Phi(\mu)<<\Phi(\nu), \mu \perp \nu \Leftrightarrow \Phi(\mu) \perp$ $\Phi(\nu)$ and similarly for $\Psi$, and $\Psi(\Phi(\mu)) \sim \mu, \Phi(\Psi(\mu)) \sim \mu$. Thus $\Phi$ induces a bijection $\widetilde{\Phi}: \mathscr{Q}_{0}(E) / \sim \rightarrow \mathscr{Q}_{0}(F) / \sim$ with inverse $\widetilde{\Psi}$. Moreover, $\widetilde{\Phi}$ maps $\mathscr{Q \mathscr { E }}_{0}(E) / \sim$ onto $\mathscr{Q}_{0}(F) / \sim$.

Proof. Fix full Borel sets $A, B$ for $E, F$ resp. and a Borel isomorphism $g$ of $E \backslash A$ with $F \mid B$. It suffices clearly to show that there is a Borel map $g: \mathscr{Q F}_{0}(E) \rightarrow \mathscr{Q S}_{0}(E \mid A)$ such that $\mu<<\nu \Leftrightarrow g(\mu)<<g(\nu), \mu \perp \nu \Leftrightarrow$ $g(\mu) \perp g(\nu)$ and similarly a Borel map $h: \mathscr{Q S}_{0}(E \mid A) \rightarrow \mathscr{Q}_{0}(E)$ with the same properties, such that $g h(\mu) \sim \mu, h g(\mu) \sim \mu$, with the further property that $g, h$ map ergodic measures to ergodic measures.

To define $g$, let $\mu \in \mathscr{Q S}_{0}(E)$. Then $\mu(A)>0$, as $A$ is full, so define a probability measure $g(\mu)$ on $A$ by $g(\mu)(B)=\mu(B) / \mu(A)$ for Borel $B \subseteq A$. It is clear that $g: \mathscr{Q S}_{0}(E) \rightarrow \mathscr{Q S}_{0}(E \mid A)$. To define $h$, let $\mu \in \mathscr{Q S}_{0}(E \mid A)$ and let $h(\mu)=\nu$ be defined as in the proof of 3.3. Note then that $g(h(\mu))=\mu$. We check next that $h g(\mu) \sim \mu$. If $\mu(B)=0$, then $\mu\left([B]_{E}\right)=0$, from which it follows that $h g(\mu)(B)=0$. If $h g(\mu)(B)=0$, then $h g(\mu)\left([B]_{E}\right)=0$, so $g(\mu)\left([B]_{E} \cap A\right)=0$, thus $\mu\left([B]_{E} \cap A\right)=0$, and, as $\left[[B]_{E} \cap A\right]_{E}=[B]_{E}$, $\mu\left([B]_{E}\right)=0$.

If $\mu<<\nu$, clearly $g(\mu)<<g(\nu)$. If $\mu<<\nu$, we verify that $h(\mu)<<h(\nu)$. If $h(\nu)(B)=0$, then (in the notation of the proof of 3.3) $\nu\left(g_{i}^{-1} \cdot\left(B \cap B_{i}\right)\right)=0$, so $\nu\left(A \cap\left[B \cap B_{i}\right]_{E}\right)=0$ (since $\left.\left[B \cap B_{i}\right]_{E}=\left[g_{i}^{-1} \cdot\left(B \cap B_{i}\right)\right]_{E}\right)$, thus $\nu\left(A \cap[B]_{E}\right)=0$, $\mu\left(A \cap[B]_{E}\right)=0$, therefore $h(\mu)(B)=0$.

Preservation of orthogonality is easy to check, as is the fact that both $g, h$ preserve ergodicity.

\section{HYPERFINITE EQUiVALENCE RELATIONS}

A countable Borel equivalence $E$ is called hyperfinite if there is an increasing sequence $E_{0} \subseteq E_{1} \subseteq E_{2} \subseteq \cdots$ of finite Borel equivalence relations with $E=$ $\bigcup_{n} E_{n}$ (i.e., $\left.x E y \Leftrightarrow \exists n\left(x E_{n} y\right)\right)$. A finite equivalence relation is one for which all equivalence classes are finite.

Remark. The condition that the sequence be increasing is crucial. Every countable Borel equivalence relation $E$ can be written as a union $E=\bigcup_{n} E_{n}$, where each $E_{n}$ is a Borel equivalence relation all of whose equivalence classes have cardinality at most 2. (This can be seen from the proof of Theorem 1.1 in [FM]: It is shown there that there is a sequence of Borel automorphisms $\left\{f_{n}\right\}$ which are of order 2 , i.e., $f_{n}^{2}=$ identity, such that $x E y \Leftrightarrow \exists n\left(f_{n}(x)=y\right)$. Put now $E_{n}=\left\{(x, y): x=y \vee f_{n}(x)=y\right\}$.)

The next result gives a series of equivalent formulations of the notion of hyperfiniteness. The equivalence of (1) and (2) is due to Weiss [W2], the direction (4) $\Rightarrow(1)$ to Weiss [W2], Slaman-Steel [SS] (see also Krieger [Kr1, 4.1.1]), and (1) $\Rightarrow$ (4) to Slaman-Steel [SS].

Theorem 5.1. Let $E$ be a countable Borel equivalence relation on $X$. Then the following are equivalent: 
(1) $E$ is hyperfinite;

(2) $E=\bigcup_{n=1}^{\infty} E_{n}$, where $E_{n}$ are finite Borel equivalence relations, $E_{n} \subseteq E_{n+1}$, and each $E_{n}$-equivalence class has cardinality at most $n$;

(3) $E=\bigcup_{n} E_{n}$, where $E_{n}$ are smooth Borel equivalence relations, $E_{n} \subseteq$ $E_{n+1}$

(4) $E=E_{\mathbb{Z}}$, i.e. there is a Borel automorphism $T$ of $\mathbb{X}$ with $x E y \Leftrightarrow \exists n \in \mathbb{Z}$ $\left(T^{n}(x)=y\right)$;

(5) There is a Borel assignment $C \mapsto<_{C}$ giving for each E-equivalence class $C$ a linear order $<_{C}$ of $C$ of order type finite or $\mathbb{Z}$. (That $C \mapsto<_{C}$ is Borel means that the relation

is Borel.)

$$
R(x, y, z): \Leftrightarrow x<_{[z]_{E}} y
$$

Proof. First note that (4), (5) are easily equivalent. Indeed, if (4) holds and assuming without loss of generality that $X=\mathbb{R}$ and letting $<$ be the usual ordering of $\mathbb{R}$, we define for each $E$-equivalence class $C$,

$$
\begin{aligned}
x<_{C} y: \Leftrightarrow x, y \in C \wedge & {[(C \text { is finite } \wedge x<y)} \\
\vee & \left.\left(C \text { is infinite } \wedge \exists n>0\left[T^{n}(x)=y\right]\right)\right] .
\end{aligned}
$$

Conversely, given $C \mapsto<_{C}$, we define $T$ by

$$
\begin{aligned}
T(x): \Leftrightarrow\left(y \text { is the successor of } x \text { in }<_{[x]_{E}}\right) \vee(x \text { is the last } \\
\text { element of } \left.<_{[x]_{E}} \text { and } y \text { is the first element }\right) .
\end{aligned}
$$

Using Theorem 1.1, it is easy to verify that $T$ is Borel. We prove now the other equivalences.

$(1) \Rightarrow(2)$. Let $E=\bigcup_{n=1}^{\infty} R_{n}, R_{n} \subseteq R_{n+1}, R_{1}=\Delta(X), R_{n}$ finite Borel equivalence relations. Define $E_{n}$ as follows: $E_{1}=R_{1}$. For any $n \geq 2$, let $X_{n}=\left\{x \in X: \operatorname{card}\left([x]_{R_{n}}\right) \leq n\right\}, X_{n-1}=\left\{x \notin X_{n}: \operatorname{card}\left([x]_{R_{n-1}}\right) \leq n\right\}$, $X_{n-2}=\left\{x \notin X_{n} \cup X_{n-1}: \operatorname{card}\left([x]_{R_{n-2}}\right) \leq n\right\}, \ldots, X_{2}=\left\{x \notin X_{n} \cup X_{n-1} \cup\right.$ $\left.\cdots \cup X_{3}: \operatorname{card}\left([x]_{R_{2}}\right) \leq n\right\}, X_{1}=X \backslash\left(X_{n} \cup X_{n-1} \cup \cdots \cup X_{2}\right)$ and put $E_{n}=R_{n} \mid$ $X_{n} \cup R_{n-1}\left|X_{n-1} \cup \cdots \cup R_{2}\right| X_{2} \cup R_{1} \mid X_{1}$.

(2) $\Rightarrow(3)$. This is clear, since any finite Borel equivalence relation is smooth.

(3) $\Rightarrow(1)$. Let $E=\bigcup_{n=0}^{\infty} E_{n}$ with $E_{n}$ smooth Borel equivalence relations, $E_{n} \subseteq E_{n+1}, E_{0}=\Delta(X)$. Let $s_{n}$ be a Borel selector for $E_{n}$, i.e., $s_{n}(x) E_{n} x$, $x E_{n} y \Rightarrow s_{n}(x)=s_{n}(y)$. Let $G_{n}=\left\{g_{n}^{(k)}\right\}_{k \in \mathbb{N}}$ be a countable group with $E_{n}=$ $E_{G_{n}}$. Define the relation $F_{n}$ on $X$ by

$$
\begin{aligned}
x F_{n} y: \Leftrightarrow \exists m \leq n\{x & E_{m} y \\
& \wedge \exists k_{0}, k_{1}, \ldots, k_{m} \\
& \leq n\left[x=g_{0}^{\left(k_{0}\right)} s_{0} g_{1}^{\left(k_{1}\right)} s_{1} \cdots g_{m}^{\left(k_{m}\right)} s_{m}(x)\right] \\
& \left.\wedge \exists l_{0}, l_{1}, \ldots, l_{m} \leq n\left[y=g_{0}^{\left(l_{0}\right)} s_{0} g_{1}^{\left(l_{1}\right)} s_{1} \cdots g_{m}^{\left(l_{m}\right)} s_{m}(y)\right]\right\} .
\end{aligned}
$$

The proof will be complete from the following claims:

(a) $F_{n} \subseteq F_{n+1}$,

(b) $F_{n} \subseteq E_{n}$,

(c) $E \subseteq \bigcup_{n} F_{n}$,

(d) $F_{n}$ is an equivalence relation,

(e) $F_{n}$ is finite. 
Claims (a), (b) are obvious. For (c), note that $\forall x \forall j \exists k_{j}(x) \quad x=g_{j}^{\left(k_{j}(x)\right.} s_{j}(x)$. If $x E y$, say $x E_{m} y$, let $n=\max \left\{m, k_{0}(x), \ldots, k_{m}(x), k_{0}(y), \ldots, k_{m}(y)\right\}$. Then $x F_{n} y$. For (e), assuming (d), note that if $x \in[y]_{F_{n}}$, there is $m \leq n$ with $x E_{m} y$ and hence $s_{m}(x)=s_{m}(y)$. So $x$ is completely determined by numbers $k_{0}, \ldots, k_{m} \leq n$ and $s_{m}(y)$, so it can only take finitely many distinct values, i.e., $[y]_{F_{n}}$ is finite.

Proof of (d). Clearly $F_{n}$ is symmetric and reflexive. We prove now that it is transitive. Assume $x F_{n} y, y F_{n} z$. Let $p, q \leq n$ be such that $x E_{p} y, y E_{q} z$ and there are $k_{0}, \ldots, k_{p}, l_{0}, \ldots, l_{r}(r=\max (p, q))$ and $m_{0}, \ldots, m_{q}$ such that $x=g_{0}^{\left(k_{0}\right)} s_{0} \cdots g_{p}^{\left(k_{p}\right)} s_{p}(x), \quad y=g_{0}^{\left(l_{0}\right)} s_{0} \cdots g_{r}^{\left(l_{r}\right)} s_{r}(y), \quad z=g_{0}^{\left(m_{0}\right)} s_{0} \cdots g_{q}^{\left(m_{q}\right)} s_{q}(z)$. Assume $p \leq q$. If $p=q$, we are done. So assume $p<q$. Let $v=$ $g_{p+1}^{\left(m_{p+1}\right)} s_{p+1} \cdots g_{q}^{\left(m_{q}\right)} s_{q}(y)$ and note that $v E_{p} y$, since $y=g_{0}^{\left(m_{0}\right)} s_{0} \cdots g_{p}^{\left(m_{p}\right)} s_{p}(v)$. Since also $x E_{p} y$, we have $s_{p}(v)=s_{p}(x)$ and so

$$
\begin{aligned}
x & =g_{0}^{\left(k_{0}\right)} s_{0} \cdots g_{p}^{\left(k_{p}\right)} s_{p}(v) \\
& =g_{0}^{\left(k_{0}\right)} s_{0} \cdots g_{p}^{\left(k_{p}\right)} s_{p} g_{p+1}^{\left(m_{p+1}\right)} s_{p+1} \cdots g_{q}^{\left(m_{q}\right)} s_{q}(y) \\
& =g_{0}^{\left(k_{0}\right)} s_{0} \cdots g_{p}^{\left(k_{p}\right)} s_{p} g_{p+1}^{\left(m_{p+1}\right)} s_{m+1} \cdots g_{q}^{\left(m_{q}\right)} s_{q}(x)
\end{aligned}
$$

since $s_{q}(x)=s_{q}(y)$. So $x F_{n} z$.

(1) $\Rightarrow(5)$. Assume $E=\bigcup_{n} E_{n}$ with $E_{n}$ increasing finite Borel equivalence relations. We will find Borel assignments $C \mapsto<_{C}^{n}$ of linear orderings to each $E_{n}$-equivalence class $C$, which are increasing, i.e., if $C=[x]_{E_{n}}, D=[x]_{E_{n+1}}$ (so that $C \subseteq D$ ) then $<_{C} \subseteq<_{D}$ (i.e. for $x, y \in C, x<_{C} y \Leftrightarrow x<_{D} y$ ) and $<_{D}$ is an end extension of $<_{C}$, i.e., for $a \in D \backslash C$, either $c<_{D} a, \forall c \in C$ or $a<_{D} c, \forall c \in C$. Then we will put, for each $E$-equivalence class $C=[x]_{E}$, $<_{C}=\bigcup_{n}<_{C_{n}}^{n}$ where $C_{n}=[x]_{E_{n}}$. Then $<_{C}$ is a linear order of $C$ and has order type finite or $\omega(=\{0,1,2, \ldots\})$ or $\omega^{*}(=\{\ldots,-2,-1,0\})$ or $\mathbb{Z}$. In the two middle cases we can easily rearrange the order in a Borel way to make it also of order type $\mathbb{Z}$, so the proof is complete, modulo the definition of $<_{C}^{n}$.

Again without loss of generality we can take $X=\mathbb{R}$ and we let $<$ be the usual order of $\mathbb{R}$. For $n=0$ let $<_{C}^{0}=<\uparrow C$. Assume now $<_{C}^{n}$ has been defined. Let $C$ be an $E_{n+1}$-equivalence class and let $C_{1}, \ldots, C_{k}$ be the $E_{n}$-equivalence classes contained in it, arranged in order so that (the <-least element of $C_{i}$ ) < (the $<$-least element of $C_{j}$ ) iff $i<j$. Then let

$$
x<_{C}^{n+1} y: \Leftrightarrow \exists k\left(x, y \in C_{k} \wedge x<_{C_{k}}^{n} y\right) \vee \exists k<l\left(x \in C_{k} \wedge y \in C_{l}\right) .
$$

$(5) \Rightarrow(1)$. Assume without loss of generality $X=2^{\mathbb{N}}$ and each $E$-equivalence class is infinite, and hence ordered by $<_{C}$ in order type $\mathbb{Z}$. For each $E$-equivalence class $C$, let $x_{C}$ be the lexicographically least element of the closure of $C$. The map $y \mapsto x_{[y]_{E}}$ is Borel. Put

$$
S_{n}^{C}=\left\{x \in C: x\left\lceil n=x_{C}\lceil n\} .\right.\right.
$$

Define now the equivalence relations $E_{n} \subseteq E$ as follows: If $(x, y) \in E$ with $[x]_{E}=[y]_{E}=C$ are such that (a) $x_{C} \in C$, then let

$$
x E_{n} y: \Leftrightarrow\left[x=y \vee \text { the distance of } x_{C} \text { from } x, y \text { in }<_{C} \text { is at most } n\right] \text {. }
$$


If (a) fails, but (b) $\exists m\left[S_{m}^{C}\right.$ is bounded below in $\left.<_{C}\right]$, let $m_{0}$ be the least such $m$ and $z_{C}$ the $<_{C}$-least element of $S_{m_{0}}^{C}$. Then put $x E_{n} y: \Leftrightarrow\left[x=y \vee\right.$ the distance of $z_{C}$ from $x, y$ in $<_{C}$ is at most $\left.n\right]$.

If (a), (b) fail but (c) $\exists m\left[S_{m}^{C}\right.$ is bounded above in $\left.<_{C}\right]$ proceed as in (b). Finally, if (a), (b), (c) fail, so that $\left\{S_{n}^{C}\right\}$ form a decreasing sequence of subsets of $C$ with $S_{n}^{C}$ unbounded in both directions in $<_{C}$ and $\bigcap_{n} S_{n}^{C}=\varnothing$, then let

$x E_{n} y: \Leftrightarrow \exists a \exists b\left[a, b\right.$ are consecutive (in $<_{C}$ ) members

$$
\text { of } S_{n}^{C} \text { and } a \leq_{C} x<_{C} b \text { and } a \leq_{C} y<_{C} b \text { ]. }
$$

It is now clear that the relations $E_{n}$ are increasing finite Borel equivalence relations with $\bigcup_{n} E_{n}=E$, so $E$ is hyperfinite.

Here are also some basic closure properties of hyperfiniteness.

Proposition 5.2. Let $E, F$ be countable Borel equivalence relations on $X, Y$ resp.

(1) $X=Y \wedge E$ is hyperfinite $\wedge F \subseteq E \Rightarrow F$ is hyperfinite;

(2) $E$ is hyperfinite $\wedge F \leq E \Rightarrow F$ is hyperfinite;

(3) $E$ is hyperfinite $\wedge A$ is Borel $\Rightarrow E \uparrow A$ is hyperfinite;

(4) $A \subseteq X$ is Borel, full for $E$ and $E \backslash A$ is hyperfinite $\Rightarrow E$ is hyperfinite;

(5) $E, F$ are hyperfinite $\Rightarrow E \times F$ is hyperfinite.

Proof. (1), (3), and (5) are immediate consequences of the definition. For (4), let $E=E_{G}$, with $G=\left\{g_{n}\right\}$ a countable group, and let for each $x \in X, N(x)=$ least $n$ with $g_{n} \cdot x \in A$. Let $E\left\lceil A=\bigcup_{n} F_{n}\right.$, with $F_{n}$ finite Borel equivalence relations on $A, F_{n} \subseteq F_{n+1}$. Define $E_{n}$ on $X$ by

$$
x E_{n} y: \Leftrightarrow\left[x E y \wedge N(x), N(y)<n \wedge g_{N(x)} \cdot x F_{n} g_{N(y)} \cdot y\right] \vee x=y .
$$

Then $E_{n} \subseteq E_{n+1}, \bigcup_{n} E_{n}=E$, and the equivalence relations $E_{n}$ are finite. Finally, for (2), let $f: Y \rightarrow X$ be a Borel map reducing $F$ to $E$, so that $f$ is countable-to-1. Then $A=f[Y] \subseteq X$ is Borel and $E \mid A$ is hyperfinite (by (3)). Let $g$ be a Borel inverse to $f$ (i.e., $g: A \rightarrow Y, f(g(x))=x$ ) and let $B=g[A]$. Then $B$ is full for $F$ and $E \uparrow A \cong F \mid B$, so $F \mid B$ is hyperfinite and so is $F$ by (4)).

Before we proceed we would like to mention some open problems:

(1) Is the increasing union of hyperfinite Borel equivalence relations hyperfinite? (It is well known [FM, 4.2] that, if $E_{n}$ are increasing Borel hyperfinite on $X$ with $E=\bigcup_{n} E_{n}$ and $\mu$ is a measure on $X$, then there is Borel $E$-invariant $A \subseteq X$ with $\mu(X \backslash A)=0$ and $E\lceil A$ hyperfinite.)

(2) Is the notion of hyperfiniteness effective? More precisely suppose $X=\mathbb{N}^{\mathbb{N}}$ and $E \in \Delta_{1}^{1}$ is a hyperfinite equivalence relation. Is there a $\Delta_{1}^{1}$ automorphism $T$ of $X$ inducing $E$ ?

(3) What is the complexity of the class of hyperfinite Borel equivalence relations, i.e. what is the complexity of the set

$\left\{x: x\right.$ codes a Borel equivalence relation on $\mathbb{N}^{\mathbb{N}}$ which is hyperfinite $\}$. It is clearly $\Sigma_{2}^{1}$. If $(2)$ has a positive answer which relativizes, then it is $\Pi_{1}^{1}$.

\section{SOME EXAMPLES}

We will discuss here some examples of hyperfinite (and nonhyperfinite) Borel equivalence relations. 
If $T$ is a Borel automorphism of a standard Borel space $X$ and we denote by $E_{T}$ the equivalence relation generated by $T$, then, by 5.1(4), $E_{T}$ is hyperfinite. Of particular importance will be the case of $T=s$, the shift on the space $2^{\mathbb{Z}}(s(x)(n)=x(n+1))$. In this case $E_{s}=E(\mathbb{Z}, 2)$ in our notation of $\S 1$. It will turn out (see $\S 9)$ that $E(\mathbb{Z}, 2)$ is invariantly universal for hyperfinite Borel $E$ (with no finite equivalence classes), i.e., for every such $E$ we have $E \sqsubseteq^{i} E(\mathbb{Z}, 2)$.

For any set $\Omega$, recall that we defined in $\S 2$ the equivalence relations $E_{0}(\Omega)$, $E_{t}(\Omega)$ on $\Omega^{\mathbb{N}}$ by

$$
\begin{gathered}
x E_{0}(\Omega) y \Leftrightarrow \exists n \forall m \geq n\left(x_{m}=y_{m}\right), \\
x E_{t}(\Omega) y \Leftrightarrow \exists n \exists m \forall k\left(x_{n+k}=y_{m+k}\right) .
\end{gathered}
$$

Then, for any countable set $\Omega, E_{0}(\Omega)$ and $E_{t}(\Omega)$ are hyperfinite. In fact, these are special cases of the following more general situation:

Let $X$ be a standard Borel space and $U$ a Borel map on $X$ such that $U$ is countable-to-1. Define the equivalence relations $E_{0}(U), E_{t}(U)$ on $X$ by

$$
\begin{gathered}
x E_{0}(U) y: \Leftrightarrow \exists n \geq 0\left[U^{n}(x)=U^{n}(y)\right] \\
x E_{t}(U) y: \Leftrightarrow \exists n \geq 0 \exists m \geq 0\left[U^{n}(x)=U^{m}(y)\right] .
\end{gathered}
$$

Then $E_{0}(\Omega), E_{t}(\Omega)$ are just $E_{0}(U), E_{t}(U)$ with $U: \Omega^{\mathbb{N}} \rightarrow \Omega^{\mathbb{N}}$ defined by $U(x)(n)=x(n+1)$ (i.e., the one-sided shift). Since $E_{0}(U)=\bigcup_{n} E_{n}$ with $x E_{n} y \Leftrightarrow U^{n}(x)=U^{n}(y)$, we see by $5.1(3)$ that $E_{0}(U)$ is hyperfinite. We will see in $\S 8$ that $E_{t}(U)$ is hyperfinite as well.

All the $E_{\mathbb{Z}}$ are hyperfinite. How about more general $E_{G}$ ? One has the following measure theoretic result.

Theorem 6.1 (Connes-Feldman-Weiss [CFW]). Let $X$ be a standard Borel space, $E$ a countable Borel equivalence relation on $X$, and $\mu$ a measure on $X$. If $E$ is of the form $E_{G}$ with $G$ a countable amenable group, or (more generally) if $E$ is $\mu$-amenable, then there is a Borel E-invariant set $Y \subseteq X$ with $\mu(X \backslash Y)=0$ such that $E \backslash Y$ is hyperfinite.

It is an open problem (see Weiss [W2]) whether every $E_{G}$ with $G$ amenable is hyperfinite. This is already open in the case $G$ is abelian, but has been proved for $G=\mathbb{Z}^{n}$ by Weiss.

There is an even stronger result in the case of category instead of measure. We need a definition first. Let $E$ be a countable Borel equivalence relation on a Polish space $X$. We call $E$ generically ergodic if every $E$-invariant Borel set is meager or comeager. We call $E$ generic if for each Borel meager set $A,[A]_{E}$ is also meager.

Theorem 6.2 (Sullivan-Weiss-Wright [SWW]). Let $X$ be a perfect Polish space and $E$ an arbitrary countable Borel equivalence relation on $X$. If $E$ is generic and generically ergodic, then there is an E-invariant dense $G_{\delta}$ set $X_{0} \subseteq X$ and there is an $E_{0}$-invariant dense $G_{\delta}$ set $Y_{0} \subseteq 2^{\mathbb{N}}$ such that $E \backslash X_{0} \cong E_{0} \mid Y_{0}$ by a continuous isomorphism. In particular, $E \uparrow X_{0}$ is hyperfinite.

The following corollary is due to Woodin.

Corollary 6.3 (Woodin). Let $E$ be a countable Borel equivalence relation on a perfect Polish space $X$. Assume $E$ is generically ergodic. Then there are a dense 
$G_{\delta} X_{0} \subseteq X$ and an $E_{0}$-invariant dense $G_{\delta} Y_{0} \subseteq 2^{\mathbb{N}}$ such that $E \backslash X_{0} \cong E_{0} \backslash Y_{0}$ by a continuous isomorphism. In particular, $E \backslash X_{0}$ is hyperfinite, and thus there is an E-invariant comeager set $Z_{0}$ (namely $\left.\left[X_{0}\right]_{E}\right)$ such that $E \backslash Z_{0}$ is hyperfinite.

Proof. Let $E=E_{G}$, with $G=\left\{g_{n}\right\}$ a countable group. Write $g_{n}(x)=g_{n} \cdot x$. Let $\left\{V_{n}\right\}$ be a basis for $X$. For each pair $(n, i) \in \mathbb{N}^{2}$ for which it is possible, choose a comeager-in- $V_{n}$ set $P_{n, i}$ such that $g_{i}\left[P_{n, i}\right]$ is meager. Put $X_{1}=$ $X \backslash \bigcup_{n, i} g_{i}\left[P_{n, i}\right]$. Then $X_{1}$ is comeager, so let $X_{2} \subseteq X_{1}$ be a dense $G_{\delta}$.

Claim. $E \backslash X_{2}$ is generically ergodic and generic.

Then, by 6.2 (since $E \backslash X_{2}$ satisfies all its conditions), there are dense $G_{\delta}$ $X_{0} \subseteq X_{2}$ and a dense $G_{\delta} E_{0}$-invariant set $Y_{0} \subseteq 2^{\mathbb{N}}$ such that $E \backslash X_{0} \cong E_{0} \mid Y_{0}$ via a continuous isomorphism. So it remains to prove the claim.

Clearly $E\left\lceil X_{2}\right.$ is generically ergodic. To show that it is generic, it is clearly enough to show that, if $B \subseteq X_{2}$ is meager in $X_{2}$ (or equivalently in $X$ ), then $[B]_{E \mid X_{2}}$ is meager in $X$. For that, note that $[B]_{E \mid X_{2}}=[B]_{E} \cap X_{2}$ and $[B]_{E}=$ $\bigcup_{i} g_{i}[B]$, so it suffices to show that $g_{i}[B] \cap X_{2}$ is meager in $X_{2}$, i.e., $g_{i}[B]$ is meager in $X$. Let $j$ be such that $g_{j}=g_{i}^{-1}$. We can of course assume that $B$ is Borel. If $g_{i}[B]$ is not meager, let $V_{n}$ be such that $g_{i}[B]$ is comeager in $V_{n}$. So there is a comeager-in- $V_{n}$ set $P$ such that $g_{i}^{-1}[P]=g_{j}[P]$ is meager. Then $P_{n, j}$ and $g_{i}[B]$ are both comeager in $V_{n}$, so they intersect. Let $x \in P_{n, j} \cap g_{i}[B]$. Then $g_{j}(x) \in B \cap g_{j}\left[P_{n, j}\right]=\varnothing$ (since $B \subseteq X_{2} \subseteq X_{1}=X \backslash \bigcup_{n, i} g_{i}\left[P_{n, i}\right]$ ).

As an application, we see immediately that, for any countable group $G$, $E(G, 2)$ is generically hyperfinite, i.e. for some Borel comeager $E$-invariant $X \subseteq 2^{G}, E \nmid X$ is hyperfinite. (On the other hand $E\left(F_{2}, 2\right)$, for example, is not hyperfinite.) For that it is enough to check that $E(G, 2)$, for infinite $G$, is generically ergodic. By 1.1 of [SWW] it is enough to see that $E(G, 2)$ has a dense orbit, and this is easy to verify using the fact that $G$ is infinite.

For another example, consider the equivalence relation $\equiv_{T}$ of Turing equivalence on $2^{\mathbb{N}}\left(x \equiv_{T} y: \Leftrightarrow x\right.$ is recursive in $y$ and $y$ is recursive in $\left.x\right)$. Again $\equiv_{T}$ is not hyperfinite (see below). However, $\equiv_{T}$ is easily generically ergodic, so $\equiv_{T}$ is generically hyperfinite.

Also, as was shown by Mycielski (see, e.g., [MU, I.6]), if $E(\mathbb{R} / \mathbb{Q})$ denotes the Vitali equivalence relation on $\mathbb{R}(x E(\mathbb{R} / \mathbb{Q}) y: \Leftrightarrow x-y \in \mathbb{Q})$ then (in our notation) $E(\mathbb{R} / \mathbb{Q}) \approx^{*} E_{0}$, so $E(\mathbb{R} / \mathbb{Q})$ is hyperfinite. This can also be seen by noticing that $E(\mathbb{R} / \mathbb{Q})$ is an increasing union of smooth Borel equivalence relations, namely $E\left(\mathbb{R} /(n !)^{-1} \mathbb{Z}\right)$. (Actually, from the results in $\S 9$ it will follow that $E(\mathbb{R} / \mathbb{Q}) \cong E_{t}$.)

Finally, in some sense, all equivalence relations induced by flows, i.e., Borel actions of $\mathbb{R}$, are "hyperfinite". More precisely, let $X$ be a standard Borel space and $g \cdot x$ a Borel action of $\mathbb{R}$ on $X$. Let $E_{\mathbb{R}}$ be the corresponding equivalence relation. Then by Wagh [Wa] (see also [K3]) $E_{\mathbb{R}}$ is Borel and there is a Borel set $A \subseteq X$ such that $A$ is full for $E_{\mathbb{R}}$ and $E_{\mathbb{R}} \mid A$ is hyperfinite. (Thus, using also [Mos, 4F.6], $E_{\mathbb{R}} \approx^{*} E_{\mathbb{R}} \backslash A$.)

We conclude this section with a couple of examples of nonhyperfinite countable Borel equivalence relations. The standard example is $E\left(F_{2}, 2\right)$ (for a proof see, e.g., [K1]). Since (identifying $2^{F_{2}}$ with $\left.2^{\mathbb{N}}\right) E\left(F_{2}, 2\right) \subseteq \equiv_{T}$, it follows that $\equiv_{T}$ is not hyperfinite as well. 


\section{THE BI-EMBEDDABILITY OF NONSMOOTH HYPERFINITE RELATIONS}

Our goal is to classify hyperfinite Borel equivalence relations up to the equivalences $\approx^{*}, \approx, \cong$. We will deal with $\approx^{*}, \approx$ here and with $\cong$ in $\S 9$.

First notice that it is easy to classify smooth relations up to $\approx^{*}, \approx$. Indeed, given a countable Borel equivalence relation $E$, let for $1 \leq n \leq \aleph_{0}$,

$$
c_{n}(E)=\operatorname{card}\left\{[x]_{E}: \operatorname{card}\left([x]_{E}\right)=n\right\} .
$$

Then we have for any smooth countable Borel $E, F$ on $X, Y$ resp.

(A) $E \approx^{*} F$ iff $\operatorname{card}(X / E)=\operatorname{card}(Y / F)$.

(B) $E \approx F$ iff $\forall n\left[\sum_{m \geq n} c_{m}(E)=\sum_{m \geq n} c_{m}(F)\right]$.

So it is enough to look at nonsmooth hyperfinite Borel equivalence relations. For those the answer is given by the next result.

Theorem 7.1. Let $E, F$ be nonsmooth hyperfinite Borel equivalence relations. Then $E \approx F$ (and thus $E \approx \approx^{*} F$ ).

Proof. It is enough, of course, to show that, for every nonsmooth hyperfinite Borel equivalence relation, we have $E \approx E_{0}$. That $E_{0} \sqsubseteq E$ follows from 3.4. We will prove below that $E \sqsubseteq E_{0}$. Recall from 1.2 that $E=E_{\mathbb{Z}} \sqsubseteq E\left(\mathbb{Z}, 2^{\mathbb{N}}\right)$. So it is enough to prove that $E\left(\mathbb{Z}, 2^{\mathbb{N}}\right) \sqsubseteq E_{0}$. Denote below $E\left(\mathbb{Z}, 2^{\mathbb{N}}\right)$ by $E$.

Call an $E$-invariant Borel set $X \subseteq\left(2^{\mathbb{N}}\right)^{\mathbb{Z}}$ smooth if $E \backslash X$ is smooth, i.e., there is a Borel selector on $X$ for $E$. We claim that it is enough to show tht $E \sqsubseteq E_{0}$ modulo smooth sets, i.e., that there is smooth $X$ such that $E$ । $\left(2^{\mathbb{N}}\right)^{\mathbb{Z}} \backslash X \bar{X} E_{0}$. Indeed let $Y=\left(2^{\mathbb{N}}\right)^{\mathbb{Z}} \backslash X$ and $f: Y \rightarrow 2^{\mathbb{N}}$ embed $E \backslash Y$ into $E_{0}$. Let $g: X \rightarrow 2^{\mathbb{N}}$ be Borel such that $x E y \Leftrightarrow g(x)=g(y)$. Also let $h: X \rightarrow \mathbb{N}$ be Borel such that $h\left\lceil[x]_{E}\right.$ is injective for any $x \in X$. Finally let $p: 2^{\mathbb{N}} \rightarrow 2^{\mathbb{N}}$ be Borel such that $x \neq y \Rightarrow \neg\left(p(x) E_{0} p(y)\right)$. Then define $F:\left(2^{\mathbb{N}}\right)^{\mathbb{Z}} \rightarrow 2^{\mathbb{N}}$ by

$$
\begin{aligned}
F(x) & =\left\langle f(x), 1^{\infty}\right\rangle, & & \text { if } x \in Y \\
& =\left\langle p g(x), 1^{h(x) \wedge} 0^{\infty}\right\rangle, & & \text { if } x \in X
\end{aligned}
$$

where for $\alpha, \beta \in 2^{\mathbb{N}},\langle\alpha, \beta\rangle \in 2^{\mathbb{N}}$ is given by

$$
\langle\alpha, \beta\rangle:=(\alpha(0), \beta(0), \alpha(1), \beta(1), \ldots)
$$

and $i^{\infty}:=(i, i, i, \ldots)$. Clearly $F$ embeds $E$ into $E_{0}$.

So in the proof below we will ignore smooth sets. Note that the collection of smooth sets is closed under countable unions.

For each $n \in \mathbb{N}, n \geq 1$, let $\left(2^{n}\right)^{n}$ be the set of all sequences $\left\langle w_{0}, \ldots, w_{n-1}\right\rangle$ with $w_{i} \in 2^{n}$, i.e., $w_{i}=\left(w_{i}(0) \cdots w_{i}(n-1)\right)$ is a binary sequence of length $n$. If $w \in\left(2^{n}\right)^{n}$ and $m \leq n$ let $w\left\lceil m\right.$ denote the sequence $\left\langle w_{0}\left\lceil m, w_{1} \mid\right.\right.$ $m, \ldots, w_{m-1}|m\rangle$. For each $n \in \mathbb{N}, n \geq 1$, fix an ordering $<_{n}$ of $\left(2^{n}\right)^{n}$ such that, given $w, v \in\left(2^{n+1}\right)^{n+1}$,

$$
w \uparrow n<_{n} v \uparrow n \Rightarrow w<_{n+1} v .
$$

For $w \in\left(2^{n}\right)^{n}$ and $x \in\left(2^{\mathbb{N}}\right)^{\mathbb{Z}}$, we say that $w$ occurs in $x$ at $k \in \mathbb{Z}$ if, for all $i<n \quad w_{i}=x_{k+i}\lceil n$. We say that $w$ occurs in $x$, in symbols $w \subseteq x$, if $w$ occurs in $x$ at some $k$. 
Note first that the set of $x \in\left(2^{\mathbb{N}}\right)^{\mathbb{Z}}$ for which there is $w \in \bigcup_{n}\left(2^{n}\right)^{n}$ occurring in $x$ but for which $\{k \in \mathbb{Z}: w$ occurs in $x$ at $k\}$ is bounded above or below is $E$-invariant and smooth, so it can be neglected, i.e., we can assume we work on the set $X \subseteq\left(2^{\mathbb{N}}\right)^{\mathbb{Z}}$ of all $x \in\left(2^{\mathbb{N}}\right)^{\mathbb{Z}}$ with the property that, whenever $w \subseteq x$, $w$ occurs in $x$ at $k$ for unboundedly many $k \in \mathbb{Z}$ in both directions.

Put now, for $x \in X$,

$$
f_{n}(x):=\text { the }<_{n} \text {-least element of }\left(2^{n}\right)^{n} \text { occurring in } x .
$$

Then note that $f_{n+1}(x) \mid n=f_{n}(x)$. Note that $x E y \Rightarrow f_{n}(x)=f_{n}(y)$. So we can define $f: X \rightarrow\left(2^{\mathbb{N}}\right)^{\mathbb{N}}$ by $f(x)=\left(u_{0}, u_{1}, \ldots\right)$ with $\left(u_{0}, u_{1}, \ldots, u_{n-1}\right)\lceil n$ $=f_{n}(x)$. Note again that $x E y \Rightarrow f(x)=f(y)$.

Given $u \in\left(2^{\mathbb{N}}\right)^{\mathbb{N}}$, we say that $u$ occurs in $x \in\left(2^{\mathbb{N}}\right)^{\mathbb{Z}}$ at $k \in \mathbb{Z}$ if $u_{i}=x_{k+i}$, $\forall i \in \mathbb{N}$, and that $u$ occurs in $x$ if it occurs in $x$ at some $k$.

The set of $x$ for which $f(x)$ occurs in $x$ and $\{k \in \mathbb{Z}: f(x)$ occurs in $x$ at $k\}$ is bounded below is clearly $E$-invariant and smooth, so it can be neglected. If $f(x)$ occurs in $x$ and $\{k \in \mathbb{Z}: f(x)$ occurs in $x$ at $k\}$ is unbounded below, then $x$ is periodic, so $[x]_{E}$ is finite. These $x$ 's form an $E$-invariant and smooth set as well and can be neglected.

So we can assume that we work on the set $Y \subseteq X$ of all $x \in X$ for which $f(x)$ does not occur in $x$. For such $x \in Y$ and $n \in \mathbb{N}$, define $k_{n}^{x} \in \mathbb{Z}$ as follows:

$$
\begin{aligned}
k_{0}^{x} & =0, \\
k_{2 n+1}^{x} & =\text { the least } k \text { such that } k>k_{2 n}^{x} \text { and } f_{2 n+1}(x) \text { occurs in } x \text { at } k, \\
k_{2 n+2}^{x} & =\text { the largest } k \text { such that } k<k_{2 n+1}^{x} \text { and } f_{2 n+2}(x) \text { occurs in } x \text { at } k .
\end{aligned}
$$

Note that if either $\left\{k_{2 n}^{x}\right\}$ or $\left\{k_{2 n+1}^{x}\right\}$ is bounded, $f(x)$ occurs in $x$. Also note that $k_{2 n+1}^{x}$ is not between $k_{2 n-1}^{x}$ and $k_{2 n}^{x}$, since this would contradict the definition of $k_{2 n}^{x}$. Similarly for $k_{2 n+2}^{x}$. So we have

$$
\cdots \leq k_{4}^{x} \leq k_{2}^{x} \leq k_{0}^{x}=0<k_{1}^{x} \leq k_{3}^{x} \leq \cdots
$$

and $k_{2 n}^{x} \rightarrow-\infty, k_{2 n+1}^{x} \rightarrow+\infty$.

Instead of working with $2^{\mathbb{N}}$ below we will work with $P(\mathbb{N})=\{A: A \subseteq \mathbb{N}\}$, identifying $A \subseteq \mathbb{N}$ with its characteristic function. Under this identification, the equivalence relation $E_{0}$ on $2^{\mathbb{N}}$ corresponds to the equivalence relation (also denoted by $\left.E_{0}\right)$ on $P(\mathbb{N})$ given by

$$
A E_{0} B: \Leftrightarrow A \triangle B \text { is finite. }
$$

Fix also a bijection $\langle\rangle:, \mathbb{N} \times \mathbb{M} \rightarrow \mathbb{N}$ where $\mathbb{M}:=$ the set of all finite sequences $\left(u_{0}, \ldots, u_{m-1}\right)$ with each $u_{i}$ a finite binary sequence.

We define now $G: Y \rightarrow P(\mathbb{N})$ as follows:

For each $n$, let $t_{n}^{x}=\left|k_{n+1}^{x}-k_{n}^{x}\right|+1$ and let $r_{n}^{x}=\left(\left(r_{n}^{x}\right)_{0}, \ldots,\left(r_{n}^{x}\right)_{m}\right)$, where $m=t_{n}^{x}-1$, be the sequence given by

$$
\left(r_{n}^{x}\right)_{i}=x_{\min \left\{k_{n}^{x}, k_{n+1}^{x}\right\}+i}\lceil n
$$

for $0 \leq i<t_{n}^{x}$. Put $G(x)=\left\{\left\langle n, r_{n}^{x}\right\rangle: n \in \mathbb{N}\right\} \subseteq \mathbb{N}$.

Note that $G$ is injective, since knowing $G(x)$ we can easily reconstruct $x$. We will show that $G$ embeds $E \backslash Y$ into $E_{0}$.

Assume $x E y$ and say, without loss of generality, $m>0$ is such that $x_{m+i}=$ $y_{i}$, for all $i$. Let $n_{0}$ be such that $k_{2 n_{0}+1}^{x}>m$. Then, since the functions 
$f_{n}$ are $E$-invariant, we have $k_{2 n_{0}+1}^{x}=m+k_{2 n_{0}+1}^{y}$, so, for all $n>2 n_{0}, k_{n}^{x}=$ $m+k_{n}^{y}$. It follows that $G(x) \triangle G(y)$ is finite, i.e., $G(x) E_{0} G(y)$. The converse, i.e., $G(x) E_{0} G(y) \Rightarrow x E y$, is even easier, and we leave it to the reader.

An immediate consequence of 7.1 and 2.3 (using the fact that $E_{t}$ is compressible and, as shown in 8.2 below, hyperfinite) is the following strengthening of 3.4 .

Corollary 7.2. Let $E$ be a countable Borel equivalence relation on a standard Borel space $X$. Then the following are equivalent:

(1) $E$ is not smooth,

(2) $E_{t} \sqsubseteq^{i} E$.

It follows also from 7.1 that, for any two nonsmooth Borel hyperfinite equivalence relations $E, F$ on $X, Y$ resp., there is a "Borel" bijection between the quotient spaces $X / E$ and $Y / F$. (Here "Borel" can be interpreted in terms of the quotient Borel structures on these quotient spaces, but of course more is true.)

Applying this in particular to $E_{0}, E(\mathbb{Z}, 2)$ provides a solution to a problem of Mycielski; see [MU, I.6].

Another corollary is that the structure of measure classes of nonatomic, quasiinvariant, (ergodic) probability measures for any nonsmooth hyperfinite Borel equivalence relation is the same (using 2.6 and 4.2).

Finally, in view of the fact that all equivalence relations induced by Borel $\mathbb{R}$-actions have full Borel sets on which their restrictions are hyperfinite (see the end of $\S 6$ ), it follows that any two nonsmooth such equivalence relations are bi-reducible. (Actually, it turns out that, if they have uncountable equivalence classes, they are Borel isomorphic.)

\section{TAIL EQUivalENCE RELATIONS}

We are going to use here ideas similar to that of the proof of 7.1 to study the tail equivalence relations $E_{t}(\Omega)$. It will be convenient to introduce a further notion here.

Let $E$ be a (not necessarily countable) Borel equivalence relation on $X$. We call $E$ hypersmooth if $E=\bigcup_{n} E_{n}, E_{n} \subseteq E_{n+1}, E_{n}$ smooth Borel equivalence relations. Thus, for countable $E$, hypersmooth = hyperfinite. Moreover, if $E \leq$ $F$ with $F$ hyperfinite, then $E$ is hypersmooth.

Consider $E_{0}\left(2^{\mathbb{N}}\right)$ (as defined in $\S 2$ ). Then easily $E_{0}\left(2^{\mathbb{N}}\right)$ is hypersmooth, but it is well known (see, e.g., [K3]) that $E_{0}\left(2^{\mathbb{N}}\right) \not \leq F$ for any countable Borel $F$. It is also easy to see that every hypersmooth $E$ is embeddable in $E_{0}\left(2^{\mathbb{N}}\right)$. Indeed, let $E=\bigcup_{n} F_{n}$ witness that $E$ is hypersmooth with $E_{0}=\Delta(X)$, and let $f_{n}: X \rightarrow 2^{\mathbb{N}}$ be such that $x F_{n} y \Leftrightarrow f_{n}(x)=f_{n}(y)$. Then, fixing a Borel isomorphism \langle\rangle$:\left(2^{\mathbb{N}}\right)^{\mathbb{N}} \rightarrow 2^{\mathbb{N}}$, the Borel map $f(x)=\left\langle f_{n}(x)\right\rangle$ embeds $E$ to $E_{0}\left(2^{\mathbb{N}}\right)$.

The following result has been proved recently by A. S. Kechris and A. Louveau (unpublished): If $E$ is Borel hypersmooth, then exactly one of the following holds: (I) $E \approx E_{0}\left(2^{\mathbb{N}}\right)$ or (II) $E \leq E_{0}$. (For results along this line in a measure theoretic context, see [Ve2].)

Consider now an arbitrary Borel map $U: X \rightarrow X$ and define the equivalence relation $E_{0}(U)$ as in $\S 6$, and similarly for the tail equivalence relation $E_{t}(U)$. 
The relations $E_{0}\left(2^{\mathbb{N}}\right), E_{t}\left(2^{\mathbb{N}}\right)$ are obtained by taking: $U:\left(2^{\mathbb{N}}\right)^{\mathbb{N}} \rightarrow\left(2^{\mathbb{N}}\right)^{\mathbb{N}}$ to be the shift $U(x)_{n}=x_{n+1}$. Clearly $E_{0}(U)$ is hypersmooth.

The next theorem extends results of Vershik [Ve1], Connes-Feldman-Weiss [CFW, Corollary 13].

Theorem 8.1. Let $U: X \rightarrow X$ be Borel. Then $E_{t}(U)$ is hypersmooth.

Corollary 8.2. If $U: X \rightarrow X$ is Borel and countable-to- 1 , then $E_{t}(U)$ is hyperfinite. In particular, $E_{t}(\Omega)$ is hyperfinite for any countable $\Omega$.

Proof. First note that $E_{t}(U) \leq E_{t}\left(2^{\mathbb{N}}\right)$. To see this, assume that $X=2^{\mathbb{N}}$ and define $f: 2^{\mathbb{N}} \rightarrow\left(2^{\mathbb{N}}\right)^{\mathbb{N}}$ by $f(x)=\left(x, U(x), U^{2}(x), \ldots\right)$. Then $f$ reduces $E_{t}(U)$ to $E_{t}\left(2^{\mathbb{N}}\right)$. So it is enough to show that $E_{t}\left(2^{\mathbb{N}}\right)$ can be reduced to $E_{0}\left(2^{\mathbb{N}}\right)$. As in the proof of 7.1, it is easy to see that we can neglect smooth sets for $E_{t}\left(2^{\mathbb{N}}\right)$.

Using again the terminology and notation of the proof of 7.1, given any $x=\left(x_{n}\right) \in\left(2^{\mathbb{N}}\right)^{\mathbb{N}}$, let

$$
s_{n}^{x}=\text { the }<_{n} \text {-least } s \in\left(2^{n}\right)^{n} \text { which occurs infinitely often in } x .
$$

Then $x E_{t}\left(2^{\mathbb{N}}\right) y \Rightarrow s_{n}^{x}=s_{n}^{y}$. Let

$$
k_{n}^{x}=\text { least } k \text { such that } s_{n}^{x} \text { occurs in } x \text { at } k \text {. }
$$

Clearly $k_{n}^{x} \leq k_{n+1}^{x}$. The set of $x$ 's for which there is $y E_{t}\left(2^{\mathbb{N}}\right) x$ with $k_{n}^{y}$ eventually constant is smooth, since, for such $x, s_{x}=\left(u_{0}, u_{1}, \ldots\right)$, where $\left(u_{0}, \ldots, u_{n-1}\right)\left\lceil n=s_{n}^{x}\right.$, is a Borel selector.

So we can work in the $E_{t}\left(2^{\mathbb{N}}\right)$-invariant set $X \subseteq\left(2^{\mathbb{N}}\right)^{\mathbb{N}}$ of $x$ 's such that, for all $y E_{t}\left(2^{\mathbb{N}}\right) x, k_{n}^{y} \rightarrow \infty$. Let \langle\rangle$: \bigcup_{n}\left(2^{\mathbb{N}}\right)^{n} \rightarrow 2^{\mathbb{N}}$ be a Borel bijection (with $\bigcup_{n}\left(2^{\mathbb{N}}\right)^{n}$ having the obvious "direct sum" Borel structure). For $x \in X$, let $\bar{\alpha}_{n}(x)=\left\langle x_{i}, x_{i+1}, \ldots, x_{j}\right\rangle$, where $i=k_{n-1}^{x}, j=k_{n}^{x}-1$, with $k_{-1}^{x}=0$, and put

$$
g(x)=\left(\bar{\alpha}_{0}(x), \bar{\alpha}_{1}(x), \ldots\right) \in\left(2^{\mathbb{N}}\right)^{\mathbb{N}} .
$$

Clearly $g$ is a Borel injection and $g(x) E_{0}\left(2^{\mathbb{N}}\right) g(y) \Rightarrow x E_{t}\left(2^{\mathbb{N}}\right) y$. Conversely, assume that $x E_{t}\left(2^{\mathbb{N}}\right) y$. Let $p, q$ be such that $x_{p+n}=y_{q+n}, \forall n \in \mathbb{N}$. Let $r$ be such that $k_{r}^{x}>p, k_{r}^{y}>q$. Then, for $t \geq r, k_{t}^{x}-p=k_{t}^{y}-q$ and so $\bar{\alpha}_{t}(x)=\bar{\alpha}_{t}(y)$ for $t>r$; therefore $g(x) E_{0}\left(2^{\mathbb{N}}\right) g(y)$.

We have just seen that $E_{t}\left(2^{\mathbb{N}}\right) \sqsubseteq E_{0}\left(2^{\mathbb{N}}\right)$. It is easy to see also that $E_{0}\left(2^{\mathbb{N}}\right) \sqsubseteq$ $E_{t}\left(2^{\mathbb{N}}\right)$. (Just send $x \in\left(2^{\mathbb{N}}\right)^{\mathbb{N}}$ to $\left(\left\langle\overline{0}, x_{0}\right\rangle,\left\langle\overline{1}, x_{1}\right\rangle, \ldots\right) \in\left(2^{\mathbb{N}}\right)^{\mathbb{N}}$ where $\bar{i}=$ $0^{i \wedge} 1^{\infty}$.) Thus $E_{0}\left(2^{\mathbb{N}}\right) \approx E_{t}\left(2^{\mathbb{N}}\right)$.

We conclude this section with an open problem concerning hypersmooth relations:

Let $E$ be a hypersmooth Borel equivalence relation on $X$ and $U: X \rightarrow X$ a Borel map, such that $x E y \Rightarrow U(x) E U(y)$. Define the equivalence relation

$$
x E_{U} y: \Leftrightarrow \exists n \geq 0 \exists m \geq 0\left(U^{n}(x) E U^{m}(y)\right) .
$$

Is $E_{U}$ hypersmooth? In particular, if $E$ is hyperfinite and $U$ is countableto-1, is $E_{U}$ hyperfinite? In [CFW], such a result is proved in the measure theoretic context.

Let us point out some consequences of an affirmative answer to this particular case of the problem. So, for the following remarks, assume that all such $E_{U}$ are hyperfinite. 
First note that, if $E_{0, U}$ is defined by

$$
x E_{0, U} y \Leftrightarrow \exists n\left[U^{n}(x) E U^{n}(x)\right],
$$

then $E_{0, U} \subseteq E_{U}$, so $E_{0, U}$ is hyperfinite as well. From this we can derive that the increasing union of a sequence of hyperfinite equivalence relations is hyperfinite (see problem (1) at the end of $\S 5$ ). Indeed, if $\left\{E_{n}\right\}$ is such a sequence on the space $X$, consider the equivalence relation $E$ on $X \times \mathbb{N}$ given by

$$
(x, n) E^{*}(y, m) \Leftrightarrow n=m \wedge x E_{n} y .
$$

Then $E^{*}$ is the "disjoint union" of the $E_{n}$, so it is hyperfinite too. Define $U$ on $X \times \mathbb{N}$ by $U(x, n)=(x, n+1)$. Then clearly $U$ is injective and $(x, n) E^{*}(y, m) \Rightarrow U(x, n) E^{*} U(y, m)$, since the $E_{n}$ are increasing. So $E_{0, U}^{*}$ is hyperfinite. But if $E=\bigcup_{n} E_{n}$, we have $x E y \Leftrightarrow(x, 0) E_{0, U}^{*}(y, 0)$, so $E$ is hyperfinite.

Further, it follows that any $E_{G}$ with $G$ countable abelian is hyperfinite (see remarks following 6.1). Indeed, $G=\bigcup G_{n}$ with $G_{n}$ finitely generated, $G_{n} \subseteq$ $G_{n+1}$, so $E_{G}=\bigcup_{n} E_{G_{n}}$, an increasing union, and thus it is enough to show, by the preceding remarks, that each $E_{G}$ with $G$ finitely generated abelian is hyperfinite. We proceed by induction on the number of generators. It is clear for one generator. Assume it is true for $n$ generators and let $G$ have $n+$ 1 generators, say $a_{1}, \ldots, a_{n+1}$. Let $H \subseteq G$ be the subgroup generated by $a_{1}, \ldots, a_{n}$ and put $E=E_{H}$. If $U$ is the automorphism corresponding to the generator $a_{n+1}$, then $x E_{H} y \Rightarrow U(x) E_{H} U(y)$ and $E_{G}=\left(E_{H}\right)_{U}$, so $E_{G}$ is hyperfinite.

\section{Classification UP TO ISOMORPHISM}

The main result of this section is the following classification theorem for aperiodic, nonsmooth hyperfinite Borel equivalence relations up to Borel isomorphism.

Theorem 9.1. Let $E, F$ be aperiodic, nonsmooth hyperfinite Borel equivalence relations. Let $\mathscr{E}_{0}(E), \mathscr{E}_{0}(F)$ be their sets of invariant, ergodic probability measures. Then

$$
E \cong F \Leftrightarrow \operatorname{card}\left(\mathscr{E}_{0}(E)\right)=\operatorname{card}\left(\mathscr{E}_{0}(F)\right) .
$$

(Note that because $E$ is aperiodic, invariant probability measures are nonatomic.)

This classification was conjectured by M. G. Nadkarni (see [CN2]), who proved first (see [N3]) the case when $\operatorname{card}\left(\mathscr{E}_{0}(E)\right)=\operatorname{card}\left(\mathscr{E}_{0}(F)\right)$ is countable, on the basis of his result (3.5 in this paper) and Theorem 7.1.

Before we proceed to the proof of 9.1 , we would like to mention some corollaries.

First, for each countable Borel equivalence relation $E$, let

$$
\begin{gathered}
c_{n}(E):=\operatorname{card}\{C \in X / E: \operatorname{card}(C)=n\}, \quad 1 \leq n \leq \aleph_{0} ; \\
s(E):=0, \quad \text { if } E \text { is smooth; } 1, \text { otherwise } \\
t(E):=\operatorname{card}\left(\mathscr{E}_{0}(E)\right) .
\end{gathered}
$$

Corollary 9.2. The sequence $\left\{\left\langle c_{n}\right\rangle_{1 \leq n \leq \aleph_{0}}, s, t\right\}$ is a complete list of invariants for Borel isomorphism of hyperfinite Borel equivalence relations.

Note that $c_{n}, t$ can take only the values $0,1,2, \ldots, \aleph_{0}, 2^{\aleph_{0}}$. 
Denote next by $\Delta(n)$ the equality relation on a set of cardinality $n, 1 \leq n \leq$ $\aleph_{0}$ and by $E^{*}(\mathbb{Z}, 2)$ the restriction of $E(\mathbb{Z}, 2)$ to its aperiodic part, i.e., to the $E(\mathbb{Z}, 2)$-invariant Borel set $\left\{x \in 2^{\mathbb{N}}: s^{n}(x) \neq x, \forall n \neq 0\right\}$, where $s$ is the shift map. Let also

$$
E \sqsubset^{i} F: \Leftrightarrow E \sqsubseteq^{i} F \wedge \neg\left(F \sqsubseteq^{i} E\right) \quad\left(\Leftrightarrow E \sqsubseteq^{i} F \wedge \neg(E \cong F)\right) .
$$

Corollary 9.3. Any aperiodic, nonsmooth hyperfinite Borel equivalence relation is Borel isomorphic to exactly one of

$$
E_{t}, E_{0} \times \Delta(n)\left(1 \leq n \leq \aleph_{0}\right), E^{*}(\mathbb{Z}, 2) .
$$

Moreover, we have

$$
E_{t} \sqsubset^{i} E_{0} \sqsubset^{i} E_{0} \times \Delta(2) \sqsubset^{i} \cdots \sqsubset^{i} E_{0} \times \Delta\left(\aleph_{0}\right) \sqsubset^{i} E^{*}(\mathbb{Z}, 2) .
$$

Proof of 9.3. Recall that $E_{t}$ is compressible, so $\mathscr{E}_{0}\left(E_{t}\right)=\varnothing$. It is easy to check that $E_{0}$ is uniquely ergodic, i.e., $\operatorname{card}\left(\mathscr{E}\left(E_{0}\right)\right)=1$, and therefore

$$
\operatorname{card}\left(\mathscr{E}_{0}\left(E_{0} \times \Delta(n)\right)\right)=n
$$

for $1 \leq n \leq \aleph_{0}$. Finally, $\operatorname{card}\left(\mathscr{E}_{0}\left(E^{*}(\mathbb{Z}, 2)\right)\right)=2^{\aleph_{0}}$ (take for example the product measures on $2^{\mathbb{Z}}$ with each coordinate having the $(p, 1-p)$-measure, $0<p<1)$. Since $\mathscr{E}_{0}(E)$ is a Borel set in a standard Borel space, $\operatorname{card}\left(\mathscr{E}_{0}(E)\right)$ is always one of $0,1,2, \ldots, \aleph_{0}, 2^{\aleph_{0}}$ so the first result follows from 9.1.

That $E_{t} \sqsubset^{i} E_{0}$ follows from 7.2, and $E_{0} \times \Delta(n) \sqsubset^{i} E_{0} \times \Delta(m)$ for $n<m$ is obvious. That $E_{0} \times \Delta\left(\aleph_{0}\right) \sqsubset^{i} E^{*}(\mathbb{Z}, 2)$ will follow immediately from the ergodic decompostion, see 9.5 below.

For the proof of Theorem 9.1 we will need two further results from ergodic theory: Dye's Theorem (see Dye [D], Sutherland [Su], Weiss [W1], or HamachiOsikawa [HO]) and the Ergodic Decomposition Theorem. Let us state first Dye's Theorem.

Dye's Theorem 9.4. Let $E, F$ be hyperfinite Borel equivalence relations on $X, Y$ resp., and let $\mu \in \mathscr{E}_{0}(E), \nu \in \mathscr{E}_{0}(F)$. Then there are invariant Borel sets $X_{0} \subseteq X, Y_{0} \subseteq Y$ such that $\mu\left(X_{0}\right)=\nu\left(Y_{0}\right)=1$ and $E\left|X_{0} \cong F\right| Y_{0}$ via $a$ Borel isomorphism that sends $\mu$ to $\nu$.

Next we will state the Ergodic Decomposition Theorem as formulated in Varadarajan [Va]. (The result actually holds, as proved in [Va], even for Borel actions of arbitrary second countable locally compact groups.)

Let $E$ be a countable Borel equivalence relation on $X$. Denote by $\mathscr{I}(E)$, $\mathscr{E}(E)$ the sets of $E$-invariant, resp. $E$-invariant ergodic, probability measures. Since $E=E_{G}$ for some Borel action of a countable group $G, \mathscr{I}(E)$ and $\mathscr{E}(E)$ are Borel sets (see [Va]). It is also proved in [Va] that $\mathscr{I}(E) \neq \varnothing$ iff $\mathscr{E}(E) \neq \varnothing$. If $E$ is aperiodic, then clearly $\mathscr{I}(E)=\mathscr{I}_{0}(E), \mathscr{E}(E)=\mathscr{E}_{0}(E)$. We have now:

Ergodic Decomposition Theorem 9.5 (Varadarajan [Va]). Let $E$ be a countable Borel equivalence relation on a standard Borel space $X$. Assume $\mathscr{I}(E) \neq \varnothing$ (thus $\mathscr{E}(E) \neq \varnothing)$. Then there is a Borel surjection $x \mapsto e_{x}$ from $X$ onto $\mathscr{E}(E)$ such that:

(1) $x E y \Rightarrow e_{x}=e_{y}$. 
(2) If $X_{e}:=\left\{x: e_{x}=e\right\}$ for any $e \in \mathscr{E}(E)$, so that $X_{e}$ is Borel and $E$ invariant by (1), then $e\left(X_{e}\right)=1$ and $e$ is the unique invariant, ergodic probability mesure for $E \uparrow X_{e}$ (i.e., $\left.\mathscr{E}\left(E \uparrow X_{e}\right)=\{e\}\right)$.

(3) For each $\mu \in \mathscr{I}(E)$, we have

$$
\mu(A)=\int e_{x}(A) d \mu(x)
$$

for $A \subseteq X$ Borel.

Proof of Theorem 9.1. We consider first the case $\operatorname{card}\left(\mathscr{E}_{0}(E)\right)=\operatorname{card}\left(\mathscr{E}_{0}(F)\right)=$ 0 . Then by Nadkarni's Theorem 3.5 and the fact that $\mathscr{I}_{0}(E)=\mathscr{J}_{0}(F)=\varnothing$, we have that $E, F$ are compressible. Then, by 7.1 and $2.3, E \cong F$.

So we can assume that $\operatorname{card}\left(\mathscr{E}_{0}(E)\right)=\operatorname{card}\left(\mathscr{E}_{0}(F)\right)>0$. Since $\mathscr{E}_{0}(E), \mathscr{E}_{0}(F)$ are Borel sets in standard Borel spaces and have the same cardinality, there is a Borel bijection $e \mapsto e^{\prime}$ from $\mathscr{E}_{0}(E)$ onto $\mathscr{E}_{0}(F)$. Let $x \mapsto e_{x}, y \mapsto f_{y}$ be ergodic decompositions of $E, F$ resp. by 9.5 . Let $X_{e}, Y_{f}$ be the corresponding sets. Then, by Dye's Theorem 9.4 , we can find invariant Borel sets $\widehat{X}_{e}, \widehat{Y}_{e^{\prime}}$ with $\widehat{X}_{e} \subseteq X_{e}, \widehat{Y}_{e^{\prime}} \subseteq Y_{e^{\prime}}, e\left(\widehat{X}_{e}\right)=e^{\prime}\left(\widehat{Y}_{e^{\prime}}\right)=1$ and Borel isomorphisms $h_{e}$ of $E \uparrow \widehat{X}_{e}, F \uparrow \widehat{Y}_{e^{\prime}}$. Since the proof of Dye's Theorem is "effective" enough, the following uniform version actually holds:

(*) If $\widehat{X}:=\bigcup_{e} \widehat{X}_{e}, \widehat{Y}:=\bigcup_{e} \widehat{Y}_{e^{\prime}}$, then $\widehat{X}, \widehat{Y}$ are Borel (and of course invariant) and also $h:=\bigcup_{e} h_{e}$ is Borel (and provides a Borel isomorphism of $E \uparrow \widehat{X}, F \uparrow \widehat{Y})$.

Granting $(*)$, we have of course that $E \uparrow \widehat{X} \cong F \mid \widehat{Y}$. Consider now $E \uparrow(X \backslash \widehat{X}), F \uparrow(Y \backslash \widehat{Y})$. By shrinking $\widehat{X}_{e_{0}}, \widehat{Y}_{e_{0}^{\prime}}$ for some fixed $e_{0}$ if necessary, we can assume that $E \uparrow(X \backslash \widehat{X}), F \uparrow(Y \backslash \widehat{Y})$ are nonsmooth. (We are using here the fact that if $R$ is a nonsmooth countable Borel equivalence relation on $Z$ and $\mu \in \mathscr{E}_{0}(R)$, then there is a Borel $R$-invariant set $W \subseteq Z$ with $\mu(W)=1$ and $R \backslash(Z \backslash W)$ nonsmooth. This follows, for example, from 7.2.) By property (3) of the ergodic decomposition, $E \backslash(X \backslash \widehat{X}), F\lceil(Y \backslash \widehat{Y})$ have no invariant probability measures, so by the argument in the beginning of the proof, $E \uparrow(X \backslash \widehat{X}) \cong F \uparrow(Y \backslash \widehat{Y})$. So $E \cong F$.

We now make some comments on the proof of $(*)$. The proof of Dye's Theorem on which it is based (see, e.g., Sutherland [Su] or Hamachi-Osikawa [HO]) proceeds by showing that, if $\lambda$ is the canonical product measure on $2^{\mathbb{N}}$ (with each coordinate having the $(1 / 2,1 / 2)$-measure), then for any hyperfinite Borel equivalence relation $E$ on $X$ and $\mu \in \mathscr{E}_{0}(E)$ there are invariant Borel sets $X_{0} \subseteq X, Y_{0} \subseteq 2^{\mathbb{N}}$ such that $\mu\left(X_{0}\right)=\lambda\left(Y_{0}\right)=1$ and $E \backslash X_{0} \cong E_{0} \mid Y_{0}$ (via a Borel isomorphism sending $\mu$ to $\lambda$-which is of course the unique element of $\left.\mathscr{E}_{0}\left(E_{0}\right)\right)$. By going in detail through the proof of Dye's Theorem, one has a parametrized version which in the case we are interested in can be formulated as follows.

Lemma 9.6. Let $E$ be an aperiodic, hyperfinite Borel equivalence relation with $\mathscr{E}_{0}(E) \neq \varnothing$. Let $x \mapsto e_{x}$ be an ergodic decomposition of $E$ with $X_{e}$ the corresponding sets. Then there are Borel invariant sets $\widetilde{X}_{e} \subseteq X_{e}, \widetilde{Y}_{e} \subseteq 2^{\mathbb{N}}$ with $e\left(\tilde{X}_{e}\right)=\lambda\left(\tilde{Y}_{e}\right)=1$ and Borel isomorphisms $g_{e}$ of $E \uparrow \widetilde{X}_{e}$ with $E_{0} \uparrow \widetilde{Y}_{e}$ sending (necessarily) $e$ to $\lambda$ such that $\tilde{X}=\bigcup_{e} X_{e}$ is Borel, and $g: \mathscr{E} \times \widetilde{X} \rightarrow 2^{\mathbb{N}}$ given by $g(e, x)=g_{e}(x)$ if $x \in \tilde{X}_{e}, 0^{\infty}$ if $x \notin \widetilde{X}_{e}$ is Borel as well. 
In earlier handwritten circulated versions of this paper we have carried out the detailed calculations needed to extract this lemma from a proof of Dye's Theorem. Mercifully, however, we found out since then that these have been written up in the literature in Krieger [Kr3], $\S 2$ and also [HO], II-4 (in somewhat different formulations), so we will refer the reader to these papers for the detailed proof.

From Lemma 9.6 it is easy to derive now $(*)$ : Let $\widetilde{X}_{e}, p_{e}$ work for $E$ and $\tilde{Y}_{f}, q_{f}$ work for $F$ according to Lemma 9.6. Put $p_{e}\left[\tilde{X}_{e}\right] \cap q_{e^{\prime}}\left[\tilde{Y}_{e^{\prime}}\right]=Z_{e} \subseteq 2^{\mathbb{N}}$, so that $Z_{e}$ is $E_{0}$-invariant and $\lambda\left(Z_{e}\right)=1$. Put $\widehat{X}_{e}=p_{e}^{-1}\left[Z_{e}\right], \widehat{Y}_{e^{\prime}}=q_{e^{\prime}}^{-1}\left[Z_{e}\right]$. Again these are invariant and $e\left(\widehat{\hat{X}}_{e}\right)=e^{\prime}\left(\widehat{Y}_{e^{\prime}}\right)=1$. Finally $h_{e}=q_{e^{\prime}}^{-1} \circ\left(p_{e} \mid \widehat{X}_{e}\right)$ is a Borel isomorphism of $E \uparrow \widehat{X}_{e}$ with $F \mid \widehat{Y}_{e^{\prime}}$. Let $\widehat{X}=\bigcup_{e} \widehat{X}_{e}, \hat{Y}=\bigcup_{e} \widehat{Y}_{e^{\prime}}$, $h=\bigcup_{e} h_{e}$. We will check that $\widehat{X}, \widehat{Y}, h$ are Borel. Take first $\widehat{X}$ ( $\hat{Y}$ being similar). We have

$$
\begin{aligned}
x \in \widehat{X} & \Leftrightarrow x \in \tilde{X} \wedge p_{e_{x}}(x) \in q_{\left(e_{x}\right)^{\prime}}\left[\widehat{Y}_{\left(e_{x}\right)^{\prime}}\right] \\
& \Leftrightarrow x \in \tilde{X} \wedge \exists y\left[f_{y}=\left(e_{x}\right)^{\prime} \wedge y \in \widetilde{Y} \wedge p\left(e_{x}, x\right)=q\left(f_{y}, y\right)\right] \\
& \Leftrightarrow x \in \tilde{X} \wedge \exists ! y\left[f_{y}=\left(e_{x}\right)^{\prime} \wedge y \in \widetilde{Y} \wedge p\left(e_{x}, x\right)=q\left(f_{y}, y\right)\right] .
\end{aligned}
$$

Since the 1-1 projection of a Borel set is Borel, $\widehat{X}$ is Borel. Finally,

$$
h(x)=y \Leftrightarrow x \in \widehat{X} \wedge y \in \widehat{Y} \wedge f_{y}=\left(e_{x}\right)^{\prime} \wedge p\left(e_{x}, x\right)=q\left(f_{y}, y\right) .
$$

So $h$ is Borel as well.

Theorem 9.1 also has the following corollary, which gives a variant of 5.1 (2):

Corollary 9.7. Let $E$ be a hyperfinite, aperiodic Borel equivalence relation on $X$. Then for any sequence $\left\{m_{n}\right\}$ with $m_{n} \geq 1, m_{n+1} / m_{n} \in \mathbb{N}, m_{n} \rightarrow \infty$, there is a sequence $\left\{E_{n}\right\}$ of finite Borel equivalence relations such that $E_{n} \subseteq E_{n+1}$, $\bigcup_{n} E_{n}=E$ and every $E_{n}$-equivalence class has cardinality $m_{n}$.

Proof. We can assume that $m_{n+1} / m_{n} \geq 2$. Identify $k_{n}$ with $\left\{0,1, \ldots, k_{n}-\right.$ $1\}$, and consider the compact product space $Y=\prod k_{n} \subseteq \mathbb{N}^{\mathbb{N}}$ and the equivalence relation $F=E_{0}(\mathbb{N}) \backslash Y$. The product measure $\mu$ on $Y$, where each $k_{n}$ has the uniform measure, is the unique invariant probability measure for $F$. If now $\operatorname{card}\left(\mathscr{E}_{0}(E)\right) \leq 1$, then $E \cong F \mid Z$, with $Z$ an $F$-invariant Borel subset of $Y$. Since the conclusion of the corollary holds trivially for $F$ (let $\left.x F_{n} y \Leftrightarrow \forall m \geq n\left(x_{m}=y_{m}\right)\right)$, it holds for $E$ as well. In the case when $\operatorname{card}\left(\mathscr{E}_{0}(E)\right)>1$, let $\Delta$ be the equality relation on a standard Borel space of cardinality equal to $\operatorname{card}\left(\mathscr{E}_{0}(E)\right)$. Then $\operatorname{card}\left(\mathscr{E}_{0}(F \times \Delta)\right)=\operatorname{card}\left(\mathscr{E}_{0}(E)\right)$, so $F \times \Delta \cong E$. Again, the conclusion of the corollary is clear for $F \times \Delta$ (using $F_{n} \times \Delta$ ), so the proof is complete.

Choosing a Borel set $X_{n}$ that meets each $E_{n}$-equivalence class at exactly one point in the above corollary, we can find a Borel automorphism $T_{n} \in[[E]]$ such that $X_{n}, T_{n}\left[X_{n}\right], \ldots, T_{n}^{m_{n}-1}\left[X_{n}\right]$ is a decomposition of $X$. Moreover, we can of course take $X_{1} \supseteq X_{2} \supseteq \cdots$, and make sure that for $x \in X_{n+1}, T_{n+1}^{k}(x)=$ $T_{n}^{k}(x)$, for $k<m_{n}$. Thus 9.7 can be viewed as a Rohlin-type lemma for aperiodic hyperfinite Borel equivalence relations. (For a discussion of the classical Rohlin lemma, see for example [W1].) 
Let us conclude this section by pointing out that Theorem 9.1, even in the case card $\left.\mathscr{E}_{0}(E)\right)=1$, can be viewed as a purely descriptive set theoretic version of Dye's Theorem (which of course was one of the key ingredients in its proof). Indeed, given $E, F, \mu, \nu$ as in Dye's Theorem 9.4, let by the Ergodic Decomposition $9.5 X_{0} \subseteq X, Y_{0} \subseteq Y$ be invariant Borel sets of measure 1 for the corresponding measures, such that $\mu$ is the only $E$-invariant measure with $\mu\left(X_{0}\right)=1$ and similarly for $Y_{0}, \nu$. Of course we can also assume that $E \uparrow X_{0}$, $F \uparrow Y_{0}$ are aperiodic. Thus, by $9.1, E \uparrow X_{0} \cong F \mid Y_{0}$. But any isomorphism must send $\mu$ to $\nu$ by the uniqueness of these measures, so we have recovered Dye's Theorem.

\section{AN EXAMPLE-LIPSCHITZ AUTOMORPHISMS OF $2^{\mathbb{N}}$}

In order to illustrate some of the ideas involved in $\S 9$, we will analyze a class of examples of hyperfinite Borel equivalence relations, namely those induced by Lipschitz automorphisms of $2^{\mathbb{N}}$.

As usual, $2^{n}$ denotes the set of binary sequences of length $n$. Given permutations $\pi, \rho$ of $2^{n}, 2^{m}$ resp. with $n \leq m$, we write $\pi \leq \rho$ if

$$
\rho(s) \uparrow n=\pi(s \uparrow n)
$$

for any $s \in 2^{m}$. If $\pi_{1} \leq \pi_{2} \leq \pi_{3} \leq \cdots$, where $\pi_{n}$ is a permutation of $2^{n}$, then $f: 2^{\mathbb{N}} \rightarrow 2^{\mathbb{N}}$ given by $f(x)=\bigcup_{n} \pi_{n}(x \mid n)$ is a homemorphism of $2^{\mathbb{N}}$. (Note that $\pi_{n}$ is uniquely determined by $f$.) These are called the Lipschitz automorphisms of $2^{\mathbb{N}}$.

We will analyze a Lipschitz automorphism $f$ in terms of its orbit tree $T_{f}$. Given $n$ and an orbit $\mathscr{O}$ of $\pi_{n}$ on $2^{n}$, say $\mathscr{O}=\left\{s_{1}, \ldots, s_{k}\right\}$ with $\pi_{n}\left(s_{i}\right)=s_{i+1}$ if $i<k$ and $\pi_{n}\left(s_{k}\right)=s_{1}$, we say that an orbit $\mathscr{O}^{\prime}$ of $\pi_{n+1}$ on $2^{n+1}$, say $\mathscr{O}^{\prime}=\left\{s_{1}^{\prime} \ldots s_{m}^{\prime}\right\}$, extends $\mathscr{O}$ if $s_{i}^{\prime} \mid n \in \mathscr{O}$ for $i=1, \ldots, m$. Then it is easy to check that either $\mathscr{O}$ has one extension $\mathscr{O}^{\prime}$ of twice the size of $\mathscr{O}$ or else $\mathscr{O}$ has two extensions $\mathscr{O}^{\prime}, \mathscr{O}^{\prime \prime}$ of the same size as $\mathscr{O}$. (In particular, every orbit $\mathscr{O}$ has size a power of 2.) The tree $T_{f}$ is the tree consisting of the orbits (of the $\pi_{n}$ 's), where the children of each orbit of $2^{n}$ are its extensions in $2^{n+1}$. More precisely, $T_{f}$ has a root (by convention). The children of the root are the ( 1 or 2 ) orbits of $2^{1}$. The children of each one of these are the (1 or 2 ) orbits of $2^{2}$ extending it, etc. We denote by $\left[T_{f}\right]$ the set of all infinite paths through $T_{f}$, i.e., $a \in\left[T_{f}\right]$ iff $a=\left(\mathscr{O}_{1}, \mathscr{O}_{2}, \ldots\right)$ where $\mathscr{O}_{n}$ is an orbit in $2^{n}$ and $\mathscr{O}_{n+1}$ extends $\mathscr{O}_{n}$.

Given any $x \in 2^{\mathbb{N}}$, denote by $a_{x}$ the unique $a \in\left[T_{f}\right]$ for which $x\lceil n \in$ $a_{x}(n), \forall n \geq 1$. Finally, for each $a \in\left[T_{f}\right]$, let

$$
X_{a}=X_{a}^{f}:=\left\{x \in 2^{\mathbb{N}}: a_{x}=a\right\} .
$$

Note that $X_{a}$ is invariant under $f$. There are two possibilities for each $X_{a}$ :

(1) From some point on, say $n_{0}$, the orbits $a(n)$ have two extensions, so $\operatorname{card}(a(n))=\operatorname{card}(a(n+1))$ for $n \geq n_{0}$. Say $\operatorname{card}\left(a\left(n_{0}\right)\right)=2^{m_{0}}$. Then $\operatorname{card}\left(X_{a}\right)=2^{m_{0}}$ and $X_{a}$ is a finite orbit of $f$.

(2) For infinitely many $n, a(n)$ has only one extension, $a(n+1)$; thus in particular $\operatorname{card}(a(n+1))=2 \operatorname{card}(a(n))$. Then $X_{a}$ is a perfect set.

In case (1), denote by $\mu_{a}=\mu_{a}^{f}$ the unique $f$-invariant probability measure living on $X_{a}$. We claim that in case (2) there is also a unique $f$-invariant 
probability measure on $X_{a}$ which we also denote by $\mu_{a}=\mu_{a}^{f}$ : Indeed, let $T_{a}$ be the tree of the closed set $X_{a}$, i.e. $\left\{s \in 2^{n}: n \in \mathbb{N}, s \in a(n)\right\}$. If $s \in T_{a} \cap 2^{n}$, define $\mu_{a}\left(N_{s}\right)=2^{-p}$, where $p=\operatorname{card}(a(n))$ and $N_{s}=\{x \in$ $\left.2^{\mathbb{N}}: x \mid n=s\right\}$. For $s \notin T_{a}$, let $\mu_{a}\left(N_{s}\right)=0$. Since clearly we have that $\mu_{a}\left(N_{s}\right)=\mu_{a}\left(N_{s^{\wedge} 0}\right)+\mu_{a}\left(N_{s^{\wedge} 1}\right)$, this defines a probability measure on $2^{\mathbb{N}}$. It is clearly $f$-invariant and easily unique.

Now put for each $x \in 2^{\mathbb{N}}, e_{x}=e_{x}^{f}:=\mu_{a_{x}}^{f}$.

Proposition 10.1. The map $x \mapsto e_{x}$ is an ergodic decomposition for $E_{f}$ (= the equivalence relation induced by $f$ ) whose components are the sets $X_{e}:=X_{a}$, where $\mu_{a}=e$.

Proof. We have to verify the properties stated in 9.5 . First we will check that $x \mapsto e_{x}$ is surjective.

Let $e \in \mathscr{E}\left(E_{f}\right)$. For each orbit $\mathscr{O}$ of some $2^{n}$, let $N_{\mathscr{O}}=\bigcup_{s \in \mathscr{O}} N_{s}$. Thus $N_{\mathscr{O}}$ is an $f$-invariant clopen set, so $e\left(N_{\mathscr{O}}\right)=0$ or 1 . So $e$ determines a unique path $a \in\left[T_{f}\right]$ with $e\left(N_{a(n)}\right)=1, \forall n$. Since $X_{a}=\bigcap_{n} N_{a(n)}$, we have $e\left(X_{a}\right)=1$, so $e=\mu_{a}$.

Clearly (1), (2) of 9.5 hold. We want now to verify (3). So let $\mu$ be $f$ invariant, $A \subseteq 2^{\mathbb{N}}$ Borel. We want to show $\mu(A)=\int \mu_{a_{x}}(A) d \mu(x)$. For that it is enough to show that $\mu\left(N_{s}\right)=\int \mu_{a_{x}}\left(N_{s}\right) d \mu(x)$ for each $s \in 2^{n}, n \in \mathbb{N}$. Now $s$ belongs to a unique orbit $\mathscr{O}$ of $2^{n}$. So clearly

$$
\mu\left(N_{s}\right)=\frac{\mu\left(N_{\mathscr{O}}\right)}{\operatorname{card}(\mathscr{O})} .
$$

Also

$$
\begin{aligned}
\int \mu_{a_{x}}\left(N_{s}\right) d \mu(x) & =\int_{N_{\mathscr{O}}} \mu_{a_{x}}\left(N_{s}\right) d \mu(x) \\
& =\int_{N_{\mathscr{O}}} \operatorname{card}(\mathscr{O})^{-1} d \mu(x)=\frac{\mu\left(N_{\mathscr{O}}\right)}{\operatorname{card}(\mathscr{O})} .
\end{aligned}
$$

Let us denote now by $C_{f}\left(2^{n}\right)$ the cardinality of the set $\left\{a \in\left[T_{f}\right]: \operatorname{card}\left(X_{a}\right)=\right.$ $\left.2^{n}\right\}$ and by $C_{f}(\infty)$ the cardinality of the set $\left\{a \in\left[T_{f}\right]: X_{a}\right.$ is perfect $\}$. Finally, for any two Borel automorphisms $f, g$ on standard Borel spaces $X, Y$ resp., let $f \cong g$ iff there is a Borel bijection $\pi: X \rightarrow Y$ such that $\pi \circ f=g \circ \pi$. We now have:

Theorem 10.2. Let $f, g$ be Lipschitz automorphsims of $2^{\mathbb{N}}$. Then the following are equivalent:

(1) $f \cong g$;

(2) $E_{f} \cong E_{g}$;

(3) $C_{f}\left(2^{n}\right)=C_{g}\left(2^{n}\right), C_{f}(\infty)=C_{g}(\infty)$ for all $n$;

(4) $f, g$ have the same cardinality of finite orbits of any given size and the same cardinality of nonatomic, ergodic, invariant probability measures.

Proof. By $10.1,(3),(4)$ are equivalent. Clearly $(1) \Rightarrow(2) \Rightarrow(4)$. We prove now that $(3) \Rightarrow(1)$.

Let $P_{f}, P_{g}$ be the sets of periodic points for $f, g$ resp. These are Borel invariant sets and by (3) $f \uparrow P_{f} \cong g \mid P_{g}$. Since $K_{f}:=\left\{a \in\left[T_{f}\right]: X_{a}^{f}\right.$ is perfect $\}$ and $K_{g}$ are Borel and have the same cardinality, there is a Borel 
bijection $a \mapsto a^{\prime}$ between them. It is thus enough to find, in a uniform Borel way from $a$, a Borel isomorphism of $f \backslash X_{a}^{f}$ with $g \backslash X_{a^{\prime}}^{g}$.

Fix $n_{0}, n_{0}^{\prime}$ such that $\operatorname{card}\left(a\left(n_{0}\right)\right)=\operatorname{card}\left(a^{\prime}\left(n_{0}^{\prime}\right)\right)=2$. Let also $\pi_{n}, \rho_{n}$ be the permutations of $2^{n}$ determining $f, g$ resp. Let $n_{0}<n_{1}<n_{2}<\cdots$ be the numbers for which card $\left(a\left(n_{k}\right)\right)=2^{k+1}$ for the first time, and similarly $n_{0}^{\prime}<$ $n_{1}^{\prime}<\cdots$ for $a^{\prime}$. By induction we can then easily define bijections $T_{k}: a\left(n_{k}\right) \rightarrow$ $a^{\prime}\left(n_{k}\right)$ such that $T_{k} \circ \pi_{n_{k}}=\rho_{n_{k}^{\prime}} \circ T_{k}$ and $T_{l}(s)\left\lceil k=T_{k}(s \backslash k)\right.$ for $l \geq k$, $s \in 2^{l}$. Then the map $x \mapsto \bigcup_{k} T_{k}\left(x \backslash n_{k}\right)$ is a Borel isomorphism of $f \backslash X_{a}^{f}$ and $g \backslash X_{a^{\prime}}^{g}$.

In the preceding result, one actually obtains a classification of Lipschitz automorphisms themselves up to Borel isomorphisms. The general problem of classifying arbitrary Borel automorphisms up to Borel isomorphism is open (see, e.g., Weiss [W2]).

It is clear that $E_{0}=E_{f}$, "except on eventually constant sequences," where $f$ has an orbit tree of the form $0-0-0 \cdots$, i.e., every orbit has only one extension. It is easy to construct orbit trees of aperiodic $f$, which have any prescribed cardinality $c_{f}(\infty) \in\left\{1,2, \ldots, \aleph_{0}, 2^{\aleph_{0}}\right\}$, and thus represent any noncompressible, aperiodic, nonsmooth hyperfinite $E$ up to Borel isomorphisin by such an $E_{f}$.

\section{Miscellanea}

We would like to collect here various remarks related to the results in this paper and other work in the literature.

(1) Let $E$ be a hyperfinite Borel equivalence relation on $X$ and $\mu \in \mathscr{Q}_{0}(E)$. Then it is well known (see e.g. Weiss [W1, p. 93] or Schmidt [S1, 8.15]) that there is $\nu \in \mathscr{Q E}_{0}\left(E_{0}\right)$ and a Borel isomorphism $\pi$ of $E \backslash X_{0}, E_{0} \backslash Y_{0}$ where $X_{0}, Y_{0}$ are invariant Borel sets with $\mu\left(X_{0}\right)=\nu\left(Y_{0}\right)=1$, such that $\pi \mu=\nu$.

This can also be seen as follows: If there is $\mu^{\prime} \sim \mu$ with $\mu^{\prime} \in \mathscr{J}_{0}(E)$, then we are done by Dye's Theorem. Otherwise, by the Hopf Theorem (see, e.g., [Fr, 3.2]), there is Borel $E$-invariant $X_{0}$ of $\mu$-measure 1 on which $E \backslash X_{0}$ is compressible. Then, by Theorem 7.1 and Proposition 2.3, $E \backslash X_{0} \cong E_{0} \backslash Y_{0}$, where $Y_{0}$ is an $E_{0}$-invariant Borel subset of $2^{\mathbb{N}}$. If $\pi$ is the Borel isomorphism, put $\nu=\pi \mu$.

(2) If $G$ is a countable group and $g \cdot x$ a Borel action of $G$ on a standard Borel space $X$, we say that the action is free if $\forall x \in X \forall g \in G(g \neq 1 \Rightarrow g \cdot x \neq$ $x)$.

There are countable Borel $E$ which cannot be represented as $E_{G}$ for a free action of a countable group $G$; see Adams [A]. However, it is not known whether, for any countable Borel $E$ and any $\mu \in \mathscr{Q}_{0}(E)$, we can write $E$ । $X=E_{G}$ for a free Borel action of a group $G$ on a Borel invariant set $X$ with $\mu(X)=1$ (ergodicity of $\mu$ is important here by the example in [A]). We also do not know whether any compressible countable Borel equivalence relation can be represented as $E_{G}$ for a free action of a countable group $G$. Also, even if a compressible $E$ can be so represented, it is not clear for what countable groups $G$ we can write $E$ as $E_{G}$ for a free action of $G$. Here is one relevant fact (whose proof uses an argument due to Mackey).

Proposition 11.1. Let $E$ be a compressible countable Borel equivalence relation. If $E=E_{G}$ for a free Borel action of a group $G$ and $G \subseteq H$ ( $H$ countable), then $E=E_{H}$ for a free Borel action of $H$. 
Proof. On the space $X \times H$ consider the following action of $G$ :

$$
g \cdot(x, h)=(g \cdot x, g h) .
$$

Denote by $\sim_{G}$ the corresponding equivalence relation. Note that $(x, h) \mapsto h$ is injective in each $\sim_{G}$-equivalence class, so $\sim_{G}$ is smooth and thus we can consider $(X \times H) / \sim_{G}$ as a standard Borel space. Consider then the following action of $H$ on $(X \times H) / \sim_{G}$ :

$$
f \cdot[(x, h)]_{\sim_{G}}=\left[\left(x, h f^{-1}\right)\right]_{\sim_{G}} .
$$

It is easy to check that it is free. Now $T(x)=[(x, 1)]_{\sim_{G}}$ is injective and $x E y \Leftrightarrow T(x) \sim_{H} T(h)$, where $\sim_{H}$ is the equivalence relation induced by the action of $H$ on $(X \times H) / \sim_{G}$. Thus $E \sqsubseteq \sim_{H}$ so, as $E$ is compressible, $E \complement^{i} \sim_{H}$ and hence $E$ is induced by a free Borel action of $H$.

In the case where $E$ is compressible hyperfinite, one can actually induce $E$ by a free action of an arbitrary infinite group.

Proposition 11.2. Let $E$ be a compressible hyperfinite Borel equivalence relation. Let $G$ be any infinite countable group. Then $E$ is of the form $E_{G}$ for a free Borel action of $G$.

Proof. Consider $E(G, 2)$ and let $X \subseteq 2^{G}$ be the free part of the action of $G$ on $2^{G}$, i.e., $X=\left\{x \in 2^{G}: \forall g \in G(g \neq 1 \Rightarrow g \cdot x \neq x)\right\}$. First it is easy to check that $X$ is a dense $G_{\delta}$. Since $E(G, 2)$ is generically ergodic (see $\S 6$ ), it follows that $E(G, 2)\lceil X$ is not smooth, so by $7.1 E \sqsubseteq E(G, 2)\lceil X$, so $E \complement^{i} E(G, 2)\lceil X$, thus $E$ can be induced by a free Borel action of $G$.

This fact provides an affirmative answer to a question mentioned in [S2, p. 16]: Does every countable infinite group have a free, nonsingular, ergodic hyperfinite action? In our terminology, this asks whether for any infinite countable $G$ there is a hyperfinite $E=E_{G}$ induced by a free Borel action of $G$, which has an ergodic, quasi-invariant measure. Since the existence of such a measure is equivalent to the nonsmoothness of $E$, this follows from 11.2.

(3) Our final remarks deal with the concept of a generator for a Borel automorphism. Given a standard Borel space $X$ and a Borel automorphism $T$ of $X$, a generator for $T$ is a partition $\left\{A_{i}\right\}_{i \in I}$ of $X$ into Borel sets such that $\left\{T^{n}\left[A_{i}\right]\right\}_{i \in I, n \in \mathbb{Z}}$ generates the Borel sets, i.e., the Borel sets form the smallest $\sigma$-algebra containing $A_{i}$ and closed under $T, T^{-1}$. Weiss [W3] has shown that every aperiodic $T$ has a countable generator (i.e. $I=\mathbb{N}$ ) modulo smooth sets (i.e., $\left\{T^{n}\left[A_{i}\right]\right\}$ generates the Borel sets modulo smooth sets), and in fact $\left\{T^{n}\left[A_{i}\right]\right\}_{i \in \mathbb{N}, n \in \mathbb{N}}$ suffices (this is sometimes called a strong generator). We can prove here (by a different method) the following:

Proposition 11.3. Let $T$ be a Borel automorphism of a standard Borel space $X$. Then $T$ has a countable generator iff $T$ has only countably many finite orbits. Proof. If $T$ has a countable generator $\left\{A_{i}\right\}_{i \in \mathbb{N}}$, then the map $\varphi: X \rightarrow \mathbb{N}^{\mathbb{Z}}$ given by $\varphi(x)(i)=n \Leftrightarrow T^{i}(x) \in A_{n}$ is a Borel injection, and if $s$ is the shift on $\mathbb{N}^{\mathbb{Z}}$ then $\varphi \circ T=s \circ \varphi$. So $\varphi$ shows that $T$ is Borel isomorphic to the restriction of $s$ onto a Borel invariant subset of $\mathbb{N}^{\mathbb{Z}}$. Since $s$ on $\mathbb{N}^{\mathbb{Z}}$ has only countably many finite orbits, so does $T$. 
Conversely, assume $T$ has only countably many finite orbits. Let $X_{0}=\{x \in$ $X$ : the orbit of $x$ is finite $\}, X_{1}=X \backslash X_{0}$. Clearly $T \uparrow X_{0}$ has a countable generator. So it is enough to show that $T \uparrow X_{1}$ has a countable generator. By 9.3, if $E_{1}=E_{T\left\lceil X_{1}\right.}$, the equivalence relation induced by $T$ on $X_{1}$, then there is a shift-invariant Borel set $Y_{1} \subseteq 2^{\mathbb{Z}}$ with $E_{1} \cong E \uparrow Y_{1}$, where $E=E(\mathbb{Z}, 2)$. Note that the shift on $2^{\mathbb{Z}}$ (and thus its restriction to $Y_{1}$ ) has a 2-generator (i.e., a generator with $I=2$ ). It will therefore be enough to prove the following lemma.

Lemma 11.4. Let $Z$ be a standard Borel space and $V$, $U$ two Borel automorphisms of $Z$. If $V, U$ induce the same equivalence relation $E$ and $U$ has a countable generator, then $V$ has a countable generator.

Proof. Partition $Z$ into Borel sets $\left\{B_{i}\right\}_{i \in \mathbb{N}}$ such that $U\left\lceil B_{i}=V^{N(i)} \uparrow B_{i}\right.$ and $U^{-1}\left\lceil B_{i}=V^{M(i)}\left\lceil B_{i}\right.\right.$, for some $N, M: \mathbb{N} \rightarrow \mathbb{N}$. Consider a generator $\left\{A_{n}\right\}_{n \in \mathbb{N}}$ for $U$ and the countable partition $\left\{C_{k}\right\}_{k \in \mathbb{N}}=\left\{B_{i} \cap A_{n}\right\}_{i, n \in \mathbb{N}}$. It is enough to show that $U^{j}\left[A_{n}\right]$ belongs to the smallest $\sigma$-algebra $\mathscr{B}$ containing all the $C_{k}$ and closed under $V, V^{-1}$. Without loss of generality, assume $j \geq 0$, and proceed by induction on $j$. For $j=0, U^{0}\left[A_{n}\right]=A_{n}=\bigcup_{i}\left(B_{i} \cap A_{n}\right)$. Assume $U^{j}\left[A_{n}\right]=B \in \mathscr{B}$. Then $U^{j+1}\left[A_{n}\right]=U[B]=U\left[\bigcup_{i}\left(B \cap B_{i}\right)\right]=\bigcup_{i} U\left[B \cap B_{i}\right]=$ $\bigcup_{i} V^{N(i)}\left[B \cap B_{i}\right]=\bigcup_{i}\left[V^{N(i)}[B] \cap \bigcup_{n} V^{N(i)}\left[B_{i} \cap A_{n}\right]\right]$.

Note that by the preceding, if $T$ is any aperiodic Borel automorphism, there is a Borel automorphism $T^{\prime}$ generating the same equivalence relation which has a 2-generator. As pointed out in Weiss [W3, p. 324], there are aperiodic $T$ which have invariant probability measures with no finite generators. But, as mentioned in the same paper, it is not known whether every $T$ with no invariant probability measures has a 2-generator.

\section{REFERENCES}

[A] S. Adams, An equivalence relation that is not freely generated, Proc. Amer. Math. Soc. 102 (1988), 565-566.

[CN1] P. Chaube and M. G. Nadkarni, $A$ version of Dye's Theorem for descriptive dynamical systems, Sankhyā Ser. A 49 (1987), 288-304.

[CN2] _ On orbit equivalence of Borel automorphisms, Proc. Indian Acad. Sci. Math. Sci. 99 (1989), 255-261.

[CFW] A. Connes, J. Feldman, and B. Weiss, An amenable equivalence relation is generated by a single transformation, Ergodic Theory Dynamical Systems 1 (1981), 431-450.

[D] H. Dye, On groups of measure preserving transformations. I, Amer. J. Math. 81 (1959), 119-159; II, ibid., 85 (1963), 551-576.

[E1] E. Effros, Transformation groups and $C^{*}$-algebras, Ann. of Math. (2) 81 (1965), 38-55.

[E2] - Polish transformation groups and classification problems (L. F. McAuley and M. M. Rao, eds.), General Topology and Modern Analysis, Academic Press, 1980, pp. 217-227.

[FM] J. Feldman and C. C. Moore, Ergodic equivalence relations, cohomology and von Neumann algebras. I, Trans. Amer. Math. Soc. 234 (1977), 289-324.

[Fr] N. Friedman, Introduction to ergodic theory, Van Nostrand, New York, 1970.

[GM] C. Graham and O. C. McGehee, Essays in commutative harmonic analysis, Springer-Verlag, New York, 1979.

[HO] T. Hamachi and M. Osikawa, Ergodic groups of automorphisms and Krieger's theorems, Sem. Math. Sci. No. 3, Keio Univ., Yokohama, 1981, 113 pp. 
[HKL] L. Harrington, A. S. Kechris, and A. Louveau, A Glimm-Effros dichotomy for Borel equivalence relations, J. Amer. Math. Soc. 3 (1990), 903-927.

[HS] E. Hewitt and K. Stromberg, Real and abstract analysis, Springer-Verlag, New York, 1969.

[Hu] W. Hurewicz, Ergodic theorem without invariant measure, Ann. of Math. (2) 45 (1944), 192-206.

[KW] Y. Katznelson and B. Weiss, The construction of quasi-invariant measures, Israel J. Math. 12 (1972), 1-4.

[K1] A. S. Kechris, Amenable equivalence relations and Turing degrees, J. Symbolic Logic 56 (1991), 182-194.

[K2] _ The structure of Borel equivalence relations in Polish spaces, Set Theory and the Continuum, (H. Judah, W. Just and W. H. Woodin, eds.), MSRI Publ., Springer-Verlag, 1992, pp. 89-102.

[K3] , Countable sections for locally compact group actions, Ergodic Theory Dynamical Systems 12 (1992), 283-295.

[KP] Yu. I. Kifer and S. A. Pirogov, The decomposition of quasi-invariant measures into ergodic components, Uspekhi Mat. Nauk 27 (1972), 239-240.

[Kr1] W. Krieger, On nonsingular transformations of a measure space. I, Z. Wahrsch. Verw. Gebiete 11 (1969), 83-97.

[Kr2] - On quasi-invariant measures in uniquely ergodic systems, Invent. Math. 14 (1971), 184-196.

[Kr3] - On ergodic flows and the isomorphism of factors, Math. Ann. 223 (1976), 19-70.

[Ku] C. Kuratowski, Topology, Vol. 1, Academic Press, New York, 1966.

[MU] D. Mauldin and S. Ulam, Mathematical problems and games, Adv. in Appl. Math. 8 (1987), 281-344.

[Mo] C. C. Moore, Ergodic theory and von Neumann algebras, Proc. Sympos. Pure Math., vol. 38, Part 2, Amer. Math. Soc., Providence, RI, 1982, pp. 179-226.

[Mos] Y. N. Moschovakis, Descriptive set theory, North-Holland, Amsterdam, 1980.

[N1] M. G. Nadkarni, Descriptive ergodic theory, Contemp. Math., vol. 94, Amer. Math. Soc., Providence, RI, 1989, pp. 191-209.

[N2] _ On the existence of a finite invariant measure, Proc. Indian Acad. Sci. Math. Sci. 100 (1991), 203-220.

[N3] _ Orbit equivalence and Kakutani equivalence in descriptive setting, reprint, 1991.

[S1] K. Schmidt, Cocycles on ergodic transformation groups, Macmillan, Delhi, 1977.

[S2] - Algebraic ideas in ergodic theory, CBMS Regional Conf. Ser. in Math., no. 76, Amer. Math. Soc., Providence, RI, 1990.

[SS] T. Slaman and J. Steel, Definable functions on degrees, Cabal Seminar 81-85, Lecture Notes in Math., vol. 1333, Springer-Verlag, 1988, pp. 37-55.

[SWW] D. Sullivan, B. Weiss, and J. D. M. Wright, Generic dynamics and monotone complete $C^{*}$-algebras, Trans. Amer. Math. Soc. 295 (1986), 795-809.

[Su] C. Sutherland, Orbit equivalence: lectures on Krieger's theorem, Univ. of Oslo Lecture Note Series, no. 23, 1976.

[Va] V. S. Varadarajan, Groups of automorphisms of Borel spaces, Trans. Amer. Math. Soc. 109 (1963), 191-220.

[Ve1] A. M. Vershik, The action of $P S L(2, \mathbb{R})$ on $P_{1} \mathbb{R}$ is approximable, Russian Math. Surveys (1) 33 (1978), 221-222.

[Ve2] _ Trajectory theory, Dynamical Systems. II (Ya. G. Sinai, eds.), Springer-Verlag, 1989, pp. 77-98.

[Wa] V. M. Wagh, A descriptive version of Ambrose's representation theorem for flows, Proc. Indian Acad. Sci. Math. Sci. 98 (1988), 101-108.

[W1] B. Weiss, Orbit equivalence of nonsingular actions, Ergod. Theory, Monographs Enseign. Math., 29, Univ. Genève, 1981, pp. 77-107. 
[W2] _ Measurable dynamics, Conf. Modern Analysis and Probability (New Haven, Conn., 1982) (R. Beals et al., eds.), Contemp. Math., vol. 26, Amer. Math. Soc., Providence, RI, 1984, pp. 395-421.

[W3] _ Countable generators in dynamics-universal minimal models, Measure and Measurable Dynamics (R. D. Mauldin et al., eds.), Contemp. Math., vol. 94, Amer. Math. Soc., Providence, RI, 1989, pp. 321-326.

Department of Mathematics, Ohio State University, Columbus, Ohio 43210

E-mail address: rld@math.ohio-state.edu

Department of Mathematics, University of North TeXas, Denton, TeXas 76203

E-mail address: jackson@untvax.bitnet

Department of Mathematics, California Institute of Technology, Pasadena, CaliFORNIA 91125

E-mail address: kechris@caltech.bitnet 